Document downloaded from:

http://hdl.handle.net/10251/51582

This paper must be cited as:

Ibáñez González, JJ.; Hernández García, V.; Arias, E.; Ruíz Martínez, PA. (2009). Solving initial value problems for ordinary differential equations by two approaches: BDF and Piecewise-linearized methods. Computer Physics Communications. 180(5):712-723. doi:10.1016/j.cpc.2008.11.013.

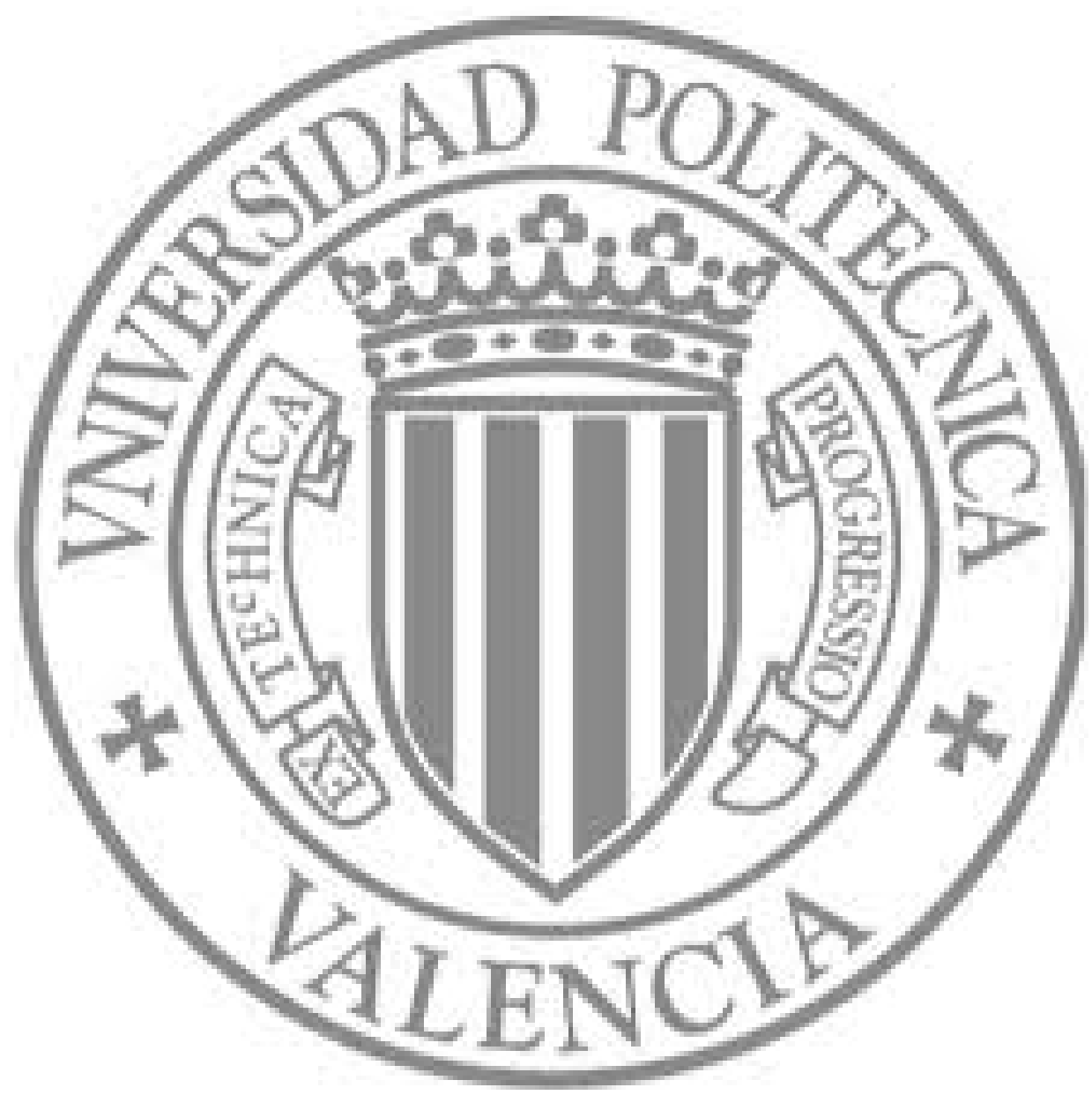

The final publication is available at

http://dx.doi.org/10.1016/j.cpc.2008.11.013

Copyright Elsevier 


\title{
Solving Initial Value Problems for Ordinary Differential Equations by two approaches: BDF and Piecewise-linearized Methods
}

\author{
J. Ibáñez ${ }^{a}$, V. Hernández ${ }^{a}$, E. Arias ${ }^{b}$, P. A. Ruiz ${ }^{a, *}$ \\ ${ }^{a}$ Instituto de Aplicaciones de las Tecnologías de la Información y de las \\ Comunicaciones Avanzadas, Technical University of Valencia, Camino de Vera \\ $s / n$, 46022-Valencia (Spain) \\ ${ }^{\mathrm{b}}$ Departamento de Sistemas Informáticos, University of Castilla-La Mancha, \\ Avda. España s/n, 02071-Albacete (Spain)
}

\begin{abstract}
Many scientific and engineering problems are described using Ordinary Differential Equations (ODEs), where the analytic solution is unknown. Much research has been done by the scientific community on developing numerical methods which can provide an approximate solution of the original ODE. In this work, two approaches have been considered based on BDF and Piecewise-linearized Methods. The approach based on BDF methods uses a Chord-Shamanskii iteration for computing the nonlinear system which is obtained when the BDF schema is used. Two approaches based on piecewise-linearized methods have also been considered. These approaches are based on a theorem proved in this paper which allows to compute the approximate solution at each time step by means of a block-oriented method based on diagonal Padé approximations. The difference between these implementations is in using or not using the scale and squaring technique.

Five algorithms based on these approaches have been developed. MATLAB and Fortran versions of the above algorithms have been developed, comparing both precision and computational costs. BLAS and LAPACK libraries have been used in Fortran implementations. In order to compare in equality of conditions all implementations, algorithms with fixed step have been considered. Four of the five case studies analyzed come from biology and chemical kinetics stiff problems. Experimental results show the advantages of the proposed algorithms, especially when they are integrating stiff problems.
\end{abstract}

Key words: Ordinary Differential Equation (ODE), Initial Value Problem (IVP), Backward Differentiation Formula (BDF) Method, Piecewise-linearized Method, Diagonal Padé Approximation, BLAS, LAPACK

PACS: 87.64.Aa 


\section{Introduction}

Much research has been done by the scientific community on developing numerical methods which permit an approximate solution to ODEs. In recent years many review articles and books have appeared on numerical methods for integrating ODEs, in particular in stiff cases [1]. Stiff problems are very common problems in many fields of the applied sciences: control theory, biology, chemical kinetics, electronic circuit theory, fluids, etc. One of the most popular multistep method families for solving IVPs for stiff ODEs is formed by the BDF methods [2-5]. Another approach used in this paper is based on piecewise-linearized methods. These methods solve an IVP by approximating the right-hand side of the ODE by means of a Taylor polynomial of degree one. The resulting approximation can be integrated analytically to obtain the solution in each subinterval and yields the exact solution for linear problems. In $[6,7]$ an exhaustive study of this approach is introduced. The developed piecewise-linearized algorithms use Theorem 1 and Corollary 1 (Section 3) which allow to compute the approximate solution at each time step by means of a block-oriented method based on diagonal Padé approximations.

The paper is structured as follows. In Section 2 a BDF algorithm is presented. The proposed approaches for solving IVPs by a piecewise-linearized method based on diagonal Padé approximations are presented in Section 3. The experimental results are shown in Section 4. Finally, some conclusions and future work are outlined in Section 5.

\section{A BDF algorithm}

\section{Let the IVP}

$$
\dot{x}(t)=f(t, x(t)), t \in\left[t_{0}, t_{f}\right], x\left(t_{0}\right)=x_{0},
$$

where $f(t, x(t)) \in \mathbb{R}^{n}, t \in\left[t_{0}, t_{f}\right]$, satisfies the necessary conditions under which the problem has a unique solution.

In this section an algorithm is presented which solves IVPs for ODEs by means of a BDF approach [8, Chapter 5] which uses a Chord-Shamanskii method [9, Chapter 5] to solve the implicit equations that appear in BDF methods. In a BDF scheme, the integration interval $\left[t_{0}, t_{f}\right]$ is divided so that the approximate solution at $t_{i}, x_{i}$, is obtained by solving an implicit nonlinear system obtained

\footnotetext{
* Corresponding author. Tel: +34-96-3877007 Ext:73538. Fax: +34-96-3877359

Email addresses: jjibanez@dsic.upv.es (J. Ibáñez), vhernand@dsic.upv.es (V. Hernández), earias@dsi.uclm.es (E. Arias), pruiz@dsic.upv.es (P. A. Ruiz ).
} 
by differentiating the polynomial which interpolates past values of $x_{i}$, and setting the derivative at $t_{i}$ to $f\left(t_{i}, x_{i}\right)$. Several methods have been implemented for solving that implicit nonlinear system; however, in the context of stiff ODEs, one of the better choices is to apply implicit schemes based on Newton's or quasi-Newton methods.

If $\left\{t_{0}, t_{1}, \cdots t_{f}\right\}$ is a partition of interval $\left[t_{0}, t_{f}\right]$ and a BDF scheme is applied, the approximate solution $x_{i}$ is obtained by solving the following equation:

$$
x_{i}-\sum_{j=1}^{r} \alpha_{j} x_{i-j}-\Delta t_{i-1} \beta f\left(t_{i}, x_{i}\right)=0,
$$

where $\Delta t_{i-1}=t_{i}-t_{i-1}$, and $\alpha_{j}(j=1,2, \cdots, r), \beta$ are parameters which appear in Table 1, where $r$ is the order of BDF method

Usually a Newton method is used to solve (2) at each time step. In this way, $x_{i}$ is obtained from the Newton iteration

$$
\begin{gathered}
x_{i}^{0}=x_{i-1}, \\
\left(I_{n}-h \beta J_{i}^{l-1}\right) \Delta x=-F\left(x_{i}^{l}\right), x_{i}^{l}=x_{i}^{l-1}+\Delta x, l \geq 1,
\end{gathered}
$$

where $J_{i}^{l-1}$ is the Jacobian matrix evaluated at $\left(x_{i}^{l-1}, t_{i}\right)$.

In this paper an inexact Newton technique for solving (3) has been used which significantly speeds up the implicit BDF integrator without loss of accuracy. This approach [9, pp. 86] uses a combination of the Chord and Shamanskii methods, based on the reduction in the nonlinear residual. This latter option decides if the Jacobian should be recomputed based on the ratio of successive residuals.

If two consecutive approaches $x_{i}^{l-1}$ and $x_{i}^{l}$ verify that $\left\|F\left(x_{i}^{l}\right)\right\| /\left\|F\left(x_{i}^{l-1}\right)\right\|$ is below a given threshold, the Jacobian is not recomputed. If the ratio is too large, the Jacobian matrix at $x_{i}^{l}$ is computed for use in the subsequent chord steps. In addition, a threshold for the ratio of successive residuals is also input. The Jacobian matrix is recomputed and factored if either the ratio of successive residuals exceeds the threshold $0<\rho<1$ or the number of iterations without an update exceeds a parameter $m \in \mathbb{N}$. Algorithm 1 (iodbcs) solves the IVP for ODEs (1) by means of the above $\underline{B}$ DF $\underline{\text { Chord- }}$ Shamanskii method.

Algorithm $1\left[\left\{x_{i}\right\}, e\right]=\operatorname{iodbcs}\left(f f, J f, x_{0}, t_{0}, t_{f}, \Delta t, r, t o l, m, \rho\right)$ Inputs: functions $f f$ and $J f$ compute $f(\tau, x) \in \mathbb{R}^{n}$ and the Jacobian matrix $J(\tau, x) \in \mathbb{R}^{n}\left(\tau \in \mathbb{R}, x \in \mathbb{R}^{n}\right)$; vector of initial conditions $x_{0} \in \mathbb{R}^{n}$; initial time $t_{0} \in \mathbb{R}$; final time $t_{f} \in \mathbb{R}$; step size $\Delta t$; order $r \in \mathbb{N}$ of the BDF method; tolerance vector tol $\in \mathbb{R}^{2}$ that contains the relative error tolerance (tol ${ }_{1}$ ) and the absolute error tolerance (tol 2 ); maximum number $m \in \mathbb{N}$ of iterations 


\begin{tabular}{|c|c|c|c|c|c|c|}
\hline & $\beta$ & $\alpha_{1}$ & $\alpha_{2}$ & $\alpha_{3}$ & $\alpha_{4}$ & $\alpha_{5}$ \\
\hline$r=1$ & 1 & 1 & & & & \\
\hline$r=2$ & $2 / 3$ & $4 / 3$ & $-1 / 3$ & & & \\
\hline$r=3$ & $6 / 11$ & $18 / 11$ & $-9 / 11$ & $2 / 11$ & & \\
\hline$r=4$ & $12 / 25$ & $48 / 25$ & $-36 / 25$ & $16 / 25$ & $-3 / 25$ & \\
\hline$r=5$ & $60 / 137$ & $300 / 137$ & $-300 / 137$ & $200 / 137$ & $-75 / 137$ & $12 / 137$ \\
\hline
\end{tabular}

Table 1

BDF method parameters (order $r=1,2,3,4$ and 5 ).

without computing the Jacobian matrix; threshold $\rho$

Outputs: Solutions $\left\{x_{i}\right\}\left(x_{i} \in \mathbb{R}^{n}\right)$ at $t_{0}, t_{0}+\Delta t, t_{0}+2 \Delta t, \ldots ; e \in \mathbb{Z}$ indicates the convergence of the method (if $e=-1$ the method does not converge)

1 Initialize $\alpha$ and $\beta$ according to the values given in Table 1

$2 i=0$

$3 m=\left\lceil\left(t_{f}-t_{0}\right) / \Delta t\right\rceil$;

4 For $i=1: m$

$4.1 t_{i}=t_{i-1}+\Delta t$

4.2 $p=\min (r, i)$

$4.3 f_{0}=\sum_{j=1}^{r} \alpha_{j} x_{i-j}$

$4.4 x_{i}=x_{i-1}$

$4.5 f=f f\left(t_{i}, x_{i}\right)$

$4.6 r_{c}=\|f\|_{\infty} ; r_{c}=r_{0}$

4.7 While $r_{c} \geq t_{1} r_{0}+$ tol $_{2}$

4.7.1 $J=J f\left(t_{i}, x_{i}\right)$

4.7.2 $[L, U]=\operatorname{lu}\left(I-\Delta t \beta_{p} J\right)$ (LU decomposition)

$4.7 .3 i_{s}=0 ; \rho=0$

4.7.4 While $i_{s}<m$ and $\rho \leq m$

4.7.4.1 $i_{s}=i_{s}+1$

4.7.4.2 Solve the lower linear system $L y=f_{0}-x_{i}+f$ for $y$

4.7.4.3 Solve the upper linear system $U \Delta x=y$ for $\Delta x$

4.7.4.4 $x_{i}=x_{i}+\Delta x$

4.7.4.5 $f=f f\left(t_{i}, x_{i}\right)$

4.7.4.6 $r_{+}=\|f\|_{\infty}$

$4.7 .4 .7 \sigma=r_{+} / r_{m} ; r_{c}=r_{+}$

4.7.4.8 If $\|\Delta x\|<$ tol $_{1} r_{0}+r_{1}$ Leave While loop 4.7.4

4.8 If $\sigma>1$

$4.8 .1 e=-1$ (The method does not converge)

4.8.2 Return 


\section{A piecewise-linearized approach for solving IVPs for ODEs}

Given a partition $t_{0}<t_{1}<\cdots<t_{l-1}<t_{l}=t_{f}$ of interval $\left[t_{0}, t_{f}\right]$, IVP (1) can be approximated by means of a set of IVPs obtained as a result of the linear approximation of $f(t, x(t))$ at each subinterval $[6,10]$

$$
\begin{gathered}
\dot{y}(t)=f_{i}+J_{i}\left(y(t)-y_{i}\right)+g_{i}\left(t-t_{i}\right), t \in\left[t_{i}, t_{i+1}\right], \\
y\left(t_{i}\right)=y_{i}, i=0,1, \cdots, l-1,
\end{gathered}
$$

where

$$
\begin{gathered}
f_{i}=f\left(t_{i}, y_{i}\right) \in \mathbb{R}^{n}, \\
J_{i}=\frac{\partial f}{\partial x}\left(t_{i}, y_{i}\right) \in \mathbb{R}^{n \times n}(\text { Jacobian matrix }), \\
\left.g_{i}=\frac{\partial f}{\partial t}\left(t_{i}, y_{i}\right) \in \mathbb{R}^{n} \text { (gradient vector }\right) .
\end{gathered}
$$

The IVP associated to the first subinterval is

$$
\begin{gathered}
\dot{y}(t)=f_{0}+J_{0}\left(y(t)-y_{0}\right)+g_{0}\left(t-t_{0}\right), t \in\left[t_{0}, t_{1}\right], \\
y\left(t_{0}\right)=y_{0}=x_{0} .
\end{gathered}
$$

Its analytic solution is given by

$$
y(t)=y_{0}+\int_{t_{0}}^{t} e^{J_{0}(t-\tau)}\left[f_{0}+g_{0}\left(\tau-t_{0}\right)\right] d \tau, t \in\left[t_{0}, t_{1}\right],
$$

therefore it is possible to compute $y_{1}=y\left(t_{1}\right)$.

By proceeding in the same way, the analytic solution of IVP associated to subinterval $i, i=1, \cdots, l-1$,

$$
\dot{y}(t)=f_{i}+J_{i}\left(y(t)-y_{i}\right)+g_{i}\left(t-t_{i}\right), t \in\left[t_{i}, t_{i+1}\right], \quad y\left(t_{i}\right)=y_{i}
$$

is given by

$$
y(t)=y_{i}+\int_{t_{i}}^{t} e^{J_{i}(t-\tau)}\left[f_{i}+g_{i}\left(\tau-t_{i}\right)\right] d \tau, \quad t \in\left[t_{i}, t_{i+1}\right]
$$

If second order partial derivatives of $f(t, x)$ are bounded on $\left[t_{0}, t_{f}\right] \times \mathbb{R}^{n}$, then the above piecewise-linearized method converges [6]. If a $(1,1)$ Padé approximation is used for computing $e^{J_{i}\left(t-t_{i}\right)}$, the above method is consistent of order 1 for autonomous ODEs and 2 for non-autonomous ODEs and linearly stable [7, pp. 26].

The piecewise-linearized approaches for solving IVPs presented in this paper are based on the following theorem and corollary. 
Theorem 1 The solution of IVP

$$
\begin{gathered}
\dot{y}(t)=f_{i}+J_{i}\left(y(t)-y_{i}\right)+g_{i}\left(t-t_{i}\right), t \in\left[t_{i}, t_{i+1}\right], \\
y\left(t_{i}\right)=y_{i} \in \mathbb{R}^{n}, \\
f_{i} \in \mathbb{R}^{n}, J_{i} \in \mathbb{R}^{n \times n}, g_{i} \in \mathbb{R}^{n},
\end{gathered}
$$

$i s$

$$
y(t)=y_{i}+F_{12}^{(i)}\left(t-t_{i}\right) f_{i}+F_{13}^{(i)}\left(t-t_{i}\right) g_{i},
$$

where $F_{12}^{(i)}\left(t-t_{i}\right)$ and $F_{13}^{(i)}\left(t-t_{i}\right)$ are the blocks $(1,2)$ and $(1,3)$ of $e^{C_{i}\left(t-t_{i}\right)}$, and

$$
C_{i}=\left[\begin{array}{ccc}
J_{i} & I_{n} & 0_{n} \\
0_{n} & 0_{n} & I_{n} \\
0_{n} & 0_{n} & 0_{n}
\end{array}\right]
$$

Proof. Defining $s=\tau-t_{i}$ and $\theta=t-t_{i}$, the integral which appears in (4) can be expressed as

$$
\int_{t_{i}}^{t} e^{J_{i}(t-\tau)}\left[f_{i}+g_{i}\left(\tau-t_{i}\right)\right] d \tau=\left[\int_{0}^{\theta} e^{J_{i}(\theta-s)} d s\right] f_{i}+\left[\int_{0}^{\theta} e^{J_{i}(\theta-s)} s d s\right] g_{i}
$$

Because $C_{i}$ is an upper-triangular block matrix, the exponential of $C_{i} \theta$ has the same structure,

$$
e^{C_{i} \theta}=\left[\begin{array}{ccc}
F_{11}^{(i)}(\theta) & F_{12}^{(i)}(\theta) & F_{13}^{(i)}(\theta) \\
0_{n} & F_{22}^{(i)}(\theta) & F_{23}^{(i)}(\theta) \\
0_{n} & 0_{n} & F_{33}^{(i)}(\theta)
\end{array}\right]
$$

where $F_{j k}^{(i)}(\theta), 1 \leq j \leq k \leq 3$, are square matrices of order $n$. Since

$$
\frac{d e^{C_{i} \theta}}{d \theta}=C_{i} e^{C_{i} \theta},\left.e^{C_{i} \theta}\right|_{\theta=0}=I_{3 n}
$$

the following IVPs are obtained 


$$
\begin{gathered}
\frac{d F_{11}^{(i)}(\theta)}{d \theta}=J_{i} F_{11}^{(i)}(\theta), F_{11}^{(i)}(0)=I_{n}, \\
\frac{d F_{22}^{(i)}(\theta)}{d \theta}=0, F_{22}^{(i)}(0)=I_{n}, \\
\frac{d F_{33}^{(i)}(\theta)}{d \theta}=0, F_{33}^{(i)}(0)=I_{n}, \\
\frac{d F_{23}^{(i)}(\theta)}{d \theta}=F_{33}^{(i)}(\theta), F_{23}^{(i)}(0)=0_{n}, \\
\frac{d F_{12}^{(i)}(\theta)}{d \theta}=J_{i} F_{12}^{(i)}(\theta)+F_{22}^{(i)}(\theta), F_{12}^{(i)}(0)=0_{n}, \\
\frac{d F_{13}^{(i)}(\theta)}{d \theta}=J_{i} F_{13}^{(i)}(\theta)+F_{23}^{(i)}(\theta), F_{13}^{(i)}(0)=0_{n} .
\end{gathered}
$$

The solutions of (8), (9) and (10) are given by

$$
\begin{gathered}
F_{11}^{(i)}(\theta)=e^{J_{i} \theta} \\
F_{22}^{(i)}(\theta)=I_{n}, \\
F_{33}^{(i)}(\theta)=I_{n} .
\end{gathered}
$$

Therefore the solutions of (11), (12) and (13) are

$$
\begin{gathered}
F_{23}^{(i)}(\theta)=\theta I_{n}, \\
F_{12}^{(i)}(\theta)=\int_{0}^{\theta} e^{J_{i}(\theta-s)} d s, \\
F_{13}^{(i)}(\theta)=\int_{0}^{\theta} e^{J_{i}(\theta-s)} s d s .
\end{gathered}
$$

Note that the integrals involved in (7) can be computed by means of (15) and (16). In conclusion, once the Jacobian matrix $J_{i}$ and the vectors $g_{i}$ and $f_{i}$ have been computed, the solution of IVP (5) is given by

$$
y(t)=y_{i}+F_{12}^{(i)}(\theta) f_{i}+F_{13}^{(i)}(\theta) g_{i}
$$

As $\theta=t-t_{i}$, then

$$
y(t)=y_{i}+F_{12}^{(i)}\left(t-t_{i}\right) f_{i}+F_{13}^{(i)}\left(t-t_{i}\right) g_{i},
$$

so the theorem is proved.

According to Theorem 1, the approximate solution of IVP (5) at $t_{i+1}$ is ob- 
tained from the approximate solution at $t_{i}$ by the following expression

$$
y_{i+1}=y_{i}+F_{12}^{(i)}\left(\Delta t_{i}\right) f_{i}+F_{13}^{(i)}\left(\Delta t_{i}\right) g_{i}
$$

where $\Delta t_{i}=t_{i+1}-t_{i}$.

For autonomous ODEs the following result is obtained.

Corollary 1 The solution of IVP

$$
\dot{y}(t)=f_{i}+J_{i}\left(y(t)-y_{i}\right), y\left(t_{i}\right)=y_{i},
$$

$i s$

$$
y(t)=y_{i}+F_{12}^{(i)}\left(t-t_{i}\right) f_{i}
$$

where $F_{12}^{(i)}\left(t-t_{i}\right)$ is the block $(1,2)$ of matrix $e^{C_{i}\left(t-t_{i}\right)}$, and

$$
C_{i}=\left[\begin{array}{ll}
J_{i} & I_{n} \\
0_{n} & 0_{n}
\end{array}\right] .
$$

Proof. It is enough to apply Theorem 1 for $g_{i}=0_{n \times 1} \in \mathbb{R}^{n}$.

According to Corollary 1, the approximate solution of IVP (19) at $t_{i+1}$ is obtained from the approximate solution at $t_{i}$ by the following expression

$$
y_{i+1}=y_{i}+F_{12}^{(i)}\left(\Delta t_{i}\right) f_{i} \text {. }
$$

Algorithm 2 is consequence of Theorem 1. This algorithm (inolex) computes the approximate solution of the IVP for non-autonomous $\underline{\text { ODEs }}$ (1) by means of a piecewise-linearized method based on the exponential of matrix $C_{i} \Delta t_{i}$.

Algorithm $2\left\{x_{i}\right\}=$ inolex $\left(\right.$ data $\left., x_{0}, t_{0}, t_{f}, \Delta t\right)$

Inputs: Data is a function that computes Jacobian matrix $J(\tau, x) \in \mathbb{R}^{n \times n}$ and function vector $f(\tau, x) \in \mathbb{R}^{n}\left(\tau \in \mathbb{R}, x \in \mathbb{R}^{n}\right)$; vector $x_{0} \in \mathbb{R}^{n}$ of initial conditions ; initial time $t_{0} \in \mathbb{R}$; final time $t_{f} \in \mathbb{R}$; step size $\Delta t \in \mathbb{R}$

Output: Vector of solutions $\left\{x_{i}\right\}\left(x_{i} \in \mathbb{R}^{n}\right)$ at $t_{0}, t_{0}+\Delta t, t_{0}+2 \Delta t, \ldots$

$1 m=\left\lceil\left(t_{f}-t_{0}\right) / \Delta t\right\rceil$

2 For $i=0: m-1$

$2.1[J, f, g]=\operatorname{data}\left(t_{i}, x_{i}\right)$

2.2 $C=\left[\begin{array}{ccc}J & I_{n} & 0_{n} \\ 0_{n} & 0_{n} & I_{n} \\ 0_{n} & 0_{n} & 0_{n}\end{array}\right]$

$2.3 F=e^{C \Delta t}$ 


\section{$2.4 t_{i+1}=t_{i}+\Delta t$}

$2.5 x_{i+1}=x_{i}+F_{12} f+F_{13} g$

\subsection{Algorithms based on the scaling and squaring technique}

The $(p, q)$ Padé approximation to $e^{A}$ is defined by

$$
R_{p q}=\left[D_{p q}(A)\right]^{-1} N_{p q}(A)
$$

where

$$
N_{p q}(A)=\sum_{k=0}^{p} \frac{(p+q-k) ! p !}{(p+k) ! k !(p-k) !} A^{k}
$$

and

$$
D_{p q}(A)=\sum_{k=0}^{p} \frac{(p+q-k) ! p !}{(p+k) ! k !(p-k) !}(-A)^{k} .
$$

Non-singularity of $D_{p q}(A)$ is assured if $p$ and $q$ are large enough or if the eigenvalues of $A$ are negative.

The problem with this method is that it only provides good approaches near the origin [11, p.573]. This problem can be avoided by using the widely used scaling and squaring method for computing the matrix exponential $[12,13]$ by exploiting the equality

$$
e^{A}=\left(e^{A / m}\right)^{m}
$$

The idea is to choose $m$ to be a power of two $\left(m=2^{j}\right)$ for which $e^{A / m}$ can be reliably and efficiently computed, and then to form the matrix $\left(e^{A / m}\right)^{m}$ by repeated squaring. One commonly used criterion for choosing $m$ is to make it the smallest power of two for which $\|A\| / m \leq 1$.

Diagonal Padé approximants $(p=q)$ are preferred, since $R_{p q}(p \neq q)$ is less accurate than $R_{l l}$, where $l=\max (p, q)$, but $R_{l l}$ can be computed at same cost.

Algorithm 3 (exmdpa) computes the exponential of a matrix by means of a scaling-squaring diagonal Padé approximation method with variable order $q$.

Algorithm $3 F=\operatorname{exmdpa}(A, q)$

Inputs: Matrix $A \in \mathbb{R}^{n \times n}$; order $q \in \mathbb{N}$ of diagonal Padé approximation of the exponential function

Output: Matrix $F=e^{A} \in \mathbb{R}^{n \times n}$

1 nor $=\|A\|_{\infty}$

$2 j_{A}=\max \left(0,1+\operatorname{int}\left(\log _{2}(\right.\right.$ nor $\left.\left.)\right)\right)$ 


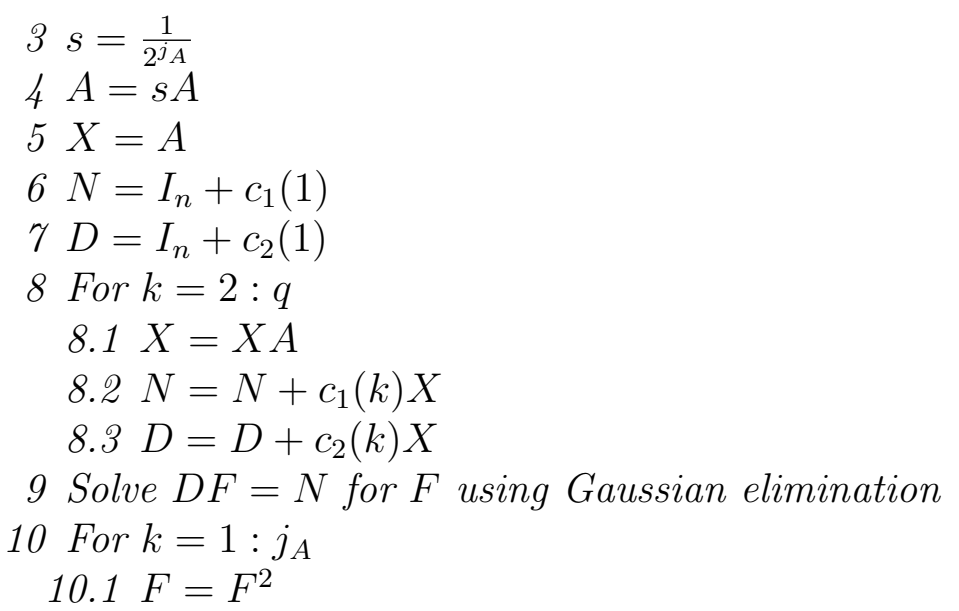

In order to reduce the high computational and storage costs of Algorithm 2, a block oriented version of Algorithm 3 has been developed. In this way, $y_{i+1}$ can be computed without explicitly computing the exponential of matrix $C \Delta t$ (step 2.3 of Algorithm 2). This algorithm only computes blocks $(1,2)$ and $(1,3)$ of the exponential of matrix

$$
A=\left[\begin{array}{lll}
J_{i} & I_{n} & 0_{n} \\
0_{n} & 0_{n} & I_{n} \\
0_{n} & 0_{n} & 0_{n}
\end{array}\right] .
$$

With this goal, some steps of Algorithm 3 are adapted to matrices

$$
\begin{gathered}
X=\left[\begin{array}{ccc}
X_{11} & X_{12} & X_{13} \\
0_{n} & X_{22} & X_{23} \\
0_{n} & 0_{n} & X_{33}
\end{array}\right], \\
N=\left[\begin{array}{ccc}
N_{11} & N_{12} & N_{13} \\
0_{n} & N_{22} & N_{23} \\
0_{n} & 0_{n} & N_{33}
\end{array}\right], \\
D=\left[\begin{array}{ccc}
D_{11} & D_{12} & D_{13} \\
0_{n} & D_{22} & D_{23} \\
0_{n} & 0_{n} & D_{33}
\end{array}\right],
\end{gathered}
$$




$$
F=\left[\begin{array}{ccc}
F_{11} & F_{12} & F_{13} \\
0_{n} & F_{22} & F_{23} \\
0_{n} & 0_{n} & F_{33}
\end{array}\right]
$$

Step 4: $A=s A$.

$$
A=s A=\left[\begin{array}{ccc}
s J_{i} & s I_{n} & 0_{n} \\
0_{n} & 0_{n} & s I_{n} \\
0_{n} & 0_{n} & 0_{n}
\end{array}\right]
$$

Let $J_{i}=s J_{i}$, then $A$ can be expressed as

$$
A=\left[\begin{array}{ccc}
J_{i} & s I_{n} & 0_{n} \\
0_{n} & 0_{n} & s I_{n} \\
0_{n} & 0_{n} & 0_{n}
\end{array}\right]
$$

Step 5: $X=A$.

$$
X=\left[\begin{array}{ccc}
J_{i} & s I_{n} & 0_{n} \\
0_{n} & 0_{n} & s I_{n} \\
0_{n} & 0_{n} & 0_{n}
\end{array}\right]
$$

Step 6: $N=I_{n}+c_{1}(1) A$.

$$
\left[\begin{array}{ccc}
N_{11} & N_{12} & N_{13} \\
0_{n} & N_{22} & N_{23} \\
0_{n} & 0_{n} & N_{33}
\end{array}\right]=\left[\begin{array}{ccc}
I_{n}+c_{1}(1) J_{i} & c_{1}(1) s I_{n} & 0_{n} \\
0_{n} & I_{n} & c_{1}(1) s I_{n} \\
0_{n} & 0_{n} & I_{n}
\end{array}\right],
$$

therefore

$$
\begin{gathered}
N_{11}=I_{n}+c_{1}(1) J_{i}, \\
N_{12}=c_{1}(1) s I_{n}, \\
N_{13}=0_{n}, \\
N_{22}=I_{n}, \\
N_{23}=c_{1}(1) s I_{n}, \\
N_{33}=I_{n} .
\end{gathered}
$$


Step 7: $D=I_{n}+c_{2}(1) A$.

$$
\left[\begin{array}{ccc}
D_{11} & D_{12} & D_{13} \\
0_{n} & D_{22} & D_{23} \\
0_{n} & 0_{n} & D_{33}
\end{array}\right]=\left[\begin{array}{ccc}
I_{n}+c_{2}(1) J_{i} & c_{2}(1) s I_{n} & 0_{n} \\
0_{n} & I_{n} & c_{2}(1) s I_{n} \\
0_{n} & 0_{n} & I_{n}
\end{array}\right]
$$

therefore

$$
\begin{gathered}
D_{11}=I_{n}+c_{2}(1) J_{i}, \\
D_{12}=c_{2}(1) s I_{n}, \\
D_{13}=0_{n}, \\
D_{22}=I_{n}, \\
D_{23}=c_{2}(1) s I_{n}, \\
D_{33}=I_{n} .
\end{gathered}
$$

Substep 8.1: $X=X A$.

$$
\left[\begin{array}{ccc}
X_{11} & X_{12} & X_{13} \\
0_{n} & 0_{n} & X_{23} \\
0_{n} & 0_{n} & 0_{n}
\end{array}\right]=\left[\begin{array}{ccc}
X_{11} & X_{12} & X_{13} \\
0_{n} & 0_{n} & X_{23} \\
0_{n} & 0_{n} & 0_{n}
\end{array}\right]\left[\begin{array}{ccc}
J_{i} & s I_{n} & 0_{n} \\
0_{n} & 0_{n} & s I_{n} \\
0_{n} & 0_{n} & 0_{n}
\end{array}\right]=\left[\begin{array}{ccc}
X_{11} J_{i} & s X_{11} & s X_{12} \\
0_{n} & 0_{n} & 0_{n} \\
0_{n} & 0_{n} & 0_{n}
\end{array}\right] .
$$

Bearing in mind data dependencies, $X_{i j}, 1 \leq i \leq j \leq 3$, can be computed as follows

$$
\begin{gathered}
X_{13}=s X_{12}, \\
X_{12}=s X_{11}, \\
X_{11}=X_{11} J_{i}, \\
X_{22}=0_{n}, \\
X_{23}=0_{n}, \\
X_{33}=0_{n} .
\end{gathered}
$$

Substep 8.2: $N=N+c_{1}(k) X$.

$$
\left[\begin{array}{ccc}
N_{11} & N_{12} & N_{13} \\
0_{n} & N_{22} & N_{23} \\
0_{n} & 0_{n} & N_{33}
\end{array}\right]=\left[\begin{array}{ccc}
N_{11}+c_{1}(k) X_{11} & N_{12}+c_{1}(k) X_{12} & N_{13}+c_{1}(k) X_{13} \\
0_{n} & N_{22} & N_{23} \\
0_{n} & 0_{n} & N_{33}
\end{array}\right] .
$$


As matrices $N_{22}, N_{23}$ and $N_{33}$ do not vary inside loop 8, then

$$
\begin{gathered}
N_{22}=I_{n}, \\
N_{23}=c_{1}(1) s I_{n}, \\
N_{33}=I_{n} .
\end{gathered}
$$

Substep 8.3: $D=D+c_{2}(k) X$.

$$
\left[\begin{array}{ccc}
D_{11} & D_{12} & D_{13} \\
0_{n} & D_{22} & D_{23} \\
0_{n} & 0_{n} & D_{33}
\end{array}\right]=\left[\begin{array}{ccc}
D_{11}+c_{2}(k) X_{11} & D_{12}+c_{2}(k) X_{12} & D_{13}+c_{2}(k) X_{13} \\
0_{n} & D_{22} & D_{23} \\
0_{n} & 0_{n} & D_{33}
\end{array}\right] .
$$

As matrices $D_{22}, D_{23}$ and $D_{33}$ do not vary inside loop 8, then

$$
\begin{gathered}
D_{22}=I_{n}, \\
D_{23}=c_{2}(1) s I_{n}, \\
D_{33}=I_{n} .
\end{gathered}
$$

Step 9: To compute $F$ by solving $D F=N$.

$$
\left[\begin{array}{ccc}
D_{11} & D_{12} & D_{13} \\
0_{n} & I_{n} & c_{2}(1) s I_{n} \\
0_{n} & 0_{n} & I_{n}
\end{array}\right]\left[\begin{array}{ccc}
F_{11} & F_{12} & F_{13} \\
0_{n} & F_{22} & F_{23} \\
0_{n} & 0_{n} & F_{33}
\end{array}\right]=\left[\begin{array}{ccc}
N_{11} & N_{12} & N_{13} \\
0_{n} & I_{n} & c_{1}(1) s I_{n} \\
0_{n} & 0_{n} & I_{n}
\end{array}\right],
$$

therefore

$$
\begin{gathered}
D_{11} F_{11}=N_{11}, \\
D_{11} F_{12}+D_{12} F_{22}=N_{12}, \\
D_{11} F_{13}+D_{12} F_{23}+D_{13} F_{33}=N_{13}, \\
F_{22}=I_{n}, \\
F_{23}+c_{2}(1) s F_{33}=c_{1}(1) s I_{n}, \\
F_{33}=I_{n} .
\end{gathered}
$$

Since $c_{1}(1)=0.5$ and $c_{2}(1)=-0.5$, then $F_{23}=s I_{n}$ and matrices $F_{11}, F_{12}$ and $F_{13}$ can be computed solving the equations

$$
\begin{gathered}
D_{11} F_{11}=N_{11}, \\
D_{11} F_{12}=N_{12}-D_{12}, \\
D_{11} F_{13}=N_{13}-s D_{12}-D_{13} .
\end{gathered}
$$


It is only necessary to know $N_{11}, N_{12}, N_{13}, D_{11}, D_{12}$ and $D_{13}$ to compute $F_{11}$, $F_{12}$ and $F_{13}$ in step 9.

Substep 10.1: $F=F^{2}$. Making the product

$$
\left[\begin{array}{ccc}
F_{11} & F_{12} & F_{13} \\
0_{n} & F_{22} & F_{23} \\
0_{n} & 0_{n} & F_{33}
\end{array}\right]=\left[\begin{array}{ccc}
F_{11} & F_{12} & F_{13} \\
0_{n} & F_{22} & F_{23} \\
0_{n} & 0_{n} & F_{33}
\end{array}\right]\left[\begin{array}{ccc}
F_{11} & F_{12} & F_{13} \\
0_{n} & F_{22} & F_{23} \\
0_{n} & 0_{n} & F_{33}
\end{array}\right],
$$

and equaling the corresponding blocks, then

$$
\begin{gathered}
F_{11}=F_{11}^{2} \\
F_{12}=F_{11} F_{12}+F_{12} F_{22}, \\
F_{13}=F_{11} F_{13}+F_{12} F_{23}+F_{13} F_{33}, \\
F_{22}=F_{22}^{2} \\
F_{23}=F_{22} F_{23}+F_{23} F_{33}, \\
F_{33}=F_{33}^{2}
\end{gathered}
$$

Bearing in mind that before entering in loop $10 F_{22}=I_{n}$ and $F_{33}=I_{n}$, then inside the above loop $F_{22}=I_{n}$ and $F_{33}=I_{n}$, therefore

$$
F_{23}=F_{23}+F_{23}=2 F_{23} \text {. }
$$

Because before entering loop $10 F_{23}=s I_{n}$, then

$$
F_{23}=2^{k} s I_{n}
$$

in the $k$ iteration. According to data dependence, $F_{11}, F_{12}$ and $F_{13}$ can be computed as

$$
\begin{gathered}
F_{13}=F_{11} F_{13}+F_{12} F_{23}+F_{13}, \\
F_{12}=F_{11} F_{12}+F_{12}, \\
F_{11}=F_{11}^{2} .
\end{gathered}
$$

The complete algorithm that solves IVP (1) corresponds to Algorithm 4 (inolsp). This algorithm solves IVPs for non-autonomous ODEs by a piecewise-linearized approach with scaling-squaring of the diagonal Padé approximants. This algorithm uses the following auxiliary algorithms:

- Algorithm 5 (coedpa) computes the coefficients of the polynomials of degree greater than zero in the diagonal Padé approximation of the exponential function.

- Algorithm 6 (inlbsp) computes the approximate solution at $t_{i+1}$ of IVP for non-autonomous ODEs (1), obtained after the piecewise-linearized process, by a block-oriented version of the scaling-squaring diagonal Padé method. 
This is the block oriented version of Algorithm 2. The approximate computational cost of Algorithm 6 is $2\left(q+3 j_{J \Delta t}+\frac{7}{3}\right) n^{3}$ flops, where $j_{J \Delta t}=$ $\max \left(0,1+\operatorname{int}\left(\log _{2}\left(\|J \Delta t\|_{\infty}\right)\right)\right)$.

Algorithm $4\left\{x_{i}\right\}=\operatorname{inolsp}\left(\right.$ data $\left., x_{0}, t_{0}, t_{f}, \Delta t, q\right)$

Inputs: Data is a function that computes function vector $f(\tau, x) \in \mathbb{R}^{n}$, Jacobian matrix $J(\tau, x) \in \mathbb{R}^{n \times n}$ and gradient vector $g(\tau, x) \in \mathbb{R}^{n}\left(\tau \in \mathbb{R}, x \in \mathbb{R}^{n}\right)$; vector $x_{0} \in \mathbb{R}^{n}$ of initial conditions; initial time $t_{0} \in \mathbb{R}$; final time $t_{f} \in \mathbb{R}$; step size $\Delta t \in \mathbb{R}$; order $q \in \mathbb{N}$ of the diagonal Padé approximation of the exponential function

Outputs: Vectors of solutions $\left\{x_{i}\right\}\left(x_{i} \in \mathbb{R}^{n}\right)$ at $t_{0}, t_{0}+\Delta t, t_{0}+2 \Delta t, \ldots$

$1\left[c_{1}, c_{2}\right]=\operatorname{coedpa}(q)$ (Algorithm 5)

$2 m=\left\lceil\left(t_{f}-t_{0}\right) / \Delta t\right\rceil$

3 For $i=0: m-1$

$3.1[f, J, g]=\operatorname{data}\left(t_{i}, x_{i}\right)$

3.2 $x_{i+1}=\operatorname{inlbsp}\left(J, f, g, x_{i}, \Delta t, c_{1}, c_{2}\right)$ (Algorithm 6)

$3.3 t_{i+1}=t_{i}+\Delta t$

Algorithm $5\left[c_{1}, c_{2}\right]=\operatorname{coedpa}(q)$

Inputs: Order $q \in \mathbb{N}$ of the diagonal Padé approximation of the exponential function

Outputs: Vectors $c_{1}, c_{2} \in \mathbb{R}^{q}$ with the coefficients of terms greater than 0 in the $(q, q)$ diagonal Padé approximation of the exponential function

$1 c_{1}(1)=0.5$

$2 c_{2}(1)=-0.5$

3 For $k=2: q$

$$
\begin{aligned}
& 3.1 c_{1}(k)=\frac{q-k+1}{(2 q-k+1) k} c_{1}(k-1) \\
& \text { 3.2 } c_{2}(k)=(-1)^{k} c_{1}(k)
\end{aligned}
$$

Algorithm $6 y_{i+1}=\operatorname{inlbsp}\left(J, f, g, y_{i}, \Delta t, c_{1}, c_{2}\right)$

Inputs: Jacobian matrix $J \in \mathbb{R}^{n \times n}$; function vector $f \in \mathbb{R}^{n}$; gradient vector $g \in \mathbb{R}^{n}$; vector $y_{i} \in \mathbb{R}^{n}$; step size $\Delta t \in \mathbb{R} ;$ vectors $c_{1}, c_{2} \in \mathbb{R}^{q}$ with the coefficients of terms of degree greater than 0 in the $(q, q)$ diagonal Padé approximation of the exponential function

Output: Vector $y_{i+1} \in \mathbb{R}^{n}$ given by expression (18)

1 nor $=\|J\|_{\infty} \Delta t$

$2 j_{J \Delta t}=\max \left(0,1+\operatorname{int}\left(\log _{2}(\right.\right.$ nor $\left.\left.)\right)\right) ; s=\frac{\Delta t}{2^{j} J \Delta t} ; J=s J$

$3 X_{11}=J ; X_{12}=s I_{n} ; X_{13}=0_{n}$

$4 N_{11}=I_{n}+c_{1}(1) J ; N_{12}=c_{1}(1) s I_{n} ; N_{13}=0_{n}$

$5 D_{11}=I_{n}+c_{2}(1) J ; D_{12}=c_{2}(2) s I_{n} ; D_{13}=0_{n}$

6 For $k=2: q$

$6.1 X_{13}=s X_{12} ; X_{12}=s X_{11} ; X_{11}=X_{11} J$ 
6.2 $N_{11}=N_{11}+c_{1}(k) X_{11} ; N_{12}=N_{12}+c_{1}(k) X_{12} ; N_{13}=N_{13}+c_{1}(k) X_{13}$

$6.3 D_{11}=D_{11}+c_{2}(k) X_{11} ; D_{12}=D_{12}+c_{2}(k) X_{12} ; D_{13}=D_{13}+c_{2}(k) X_{13}$

7 Solve $D_{11} F_{11}=N_{11}$ for $F_{11}$

8 Solve $D_{11} F_{12}=N_{12}-D_{12}$ for $F_{12}$

9 Solve $D_{11} F_{13}=N_{13}-s D_{12}-D_{13}$ for $F_{13}$

10 For $k=1: j_{J \Delta t}$

$10.1 F_{13}=F_{11} F_{13}+s F_{12}+F_{13} ; F_{12}=F_{11} F_{12}+F_{12} ; F_{11}=F_{11}^{2}$

10.2 $s=2 s$

$11 y_{i+1}=y_{i}+F_{12} f+F_{13} g$

For autonomous ODEs the computational and storage costs can be reduced if Corollary 1 is applied. Hence, another algorithm (iaolsp) can be developed to solve IVPs for autonomous ODEs by a piecewise-linearized approach with scaling-squaring of the diagonal Padé approximants.

This algorithm uses the auxiliary Algorithms 5 (coedpa) and 8. Algorithm 8 (ialbsp) computes the approximate solution at $t_{i+1}$ of IVP for autonomous ODEs (1), obtained after the piecewise-linearized process, by a block-oriented version of the scaling-squaring diagonal Padé method. The approximate computational cost of Algorithm 8 is $2\left(q+2 j_{J \Delta t}+\frac{4}{3}\right) n^{3}$ flops, where $j_{J \Delta t}=$ $\max \left(0,1+\operatorname{int}\left(\log _{2}\left(\|J \Delta t\|_{\infty}\right)\right)\right)$.

Algorithm $7\left\{x_{i}\right\}=$ iaolsp $\left(\right.$ data $\left., x_{0}, t_{0}, t_{f}, \Delta t, q\right)$

Inputs: Data is a function that computes function vector $f(\tau, x) \in \mathbb{R}^{n}$ and the Jacobian matrix $J(\tau, x) \in \mathbb{R}^{n \times n}$; vector $x_{0} \in \mathbb{R}^{n}$ of initial conditions; initial time $t_{0} \in \mathbb{R} ;$ final time $t_{f} \in \mathbb{R}$; step size $\Delta t \in \mathbb{R}$; order $q \in \mathbb{N}$ of the diagonal Padé approximation of the exponential function

Outputs: Vectors of solutions $\left\{x_{i}\right\}\left(x_{i} \in \mathbb{R}^{n}\right)$ at $t_{0}, t_{0}+\Delta t, t_{0}+2 \Delta t, \ldots$

$1\left[c_{1}, c_{2}\right]=\operatorname{coedpa}(q)$ (Algorithm 5)

$2 m=\left\lceil\left(t_{f}-t_{0}\right) / \Delta t\right\rceil$

3 For $i=0: m-1$

$3.1[f, J, g]=\operatorname{data}\left(t_{i}, x_{i}\right)$

3.2 $x_{i+1}=\operatorname{ialbsp}\left(J, f, x_{i}, \Delta t, c_{1}, c_{2}\right)$ (Algorithm 8)

$3.3 t_{i+1}=t_{i}+\Delta t$

Algorithm $8 y_{i+1}=i a l b s p\left(J, f, y_{i}, \Delta t, c_{1}, c_{2}\right)$

Inputs: Jacobian matrix $J \in \mathbb{R}^{n \times n}$; function vector $f \in \mathbb{R}^{n}$; vector $y_{i} \in \mathbb{R}^{n}$; step size $\Delta t \in \mathbb{R}$; vectors $c_{1}, c_{2} \in \mathbb{R}^{q}$ with the coefficients of terms of degree greater than 0 in the $(q, q)$ diagonal Padé approximation of the exponential function

Output: Vector $y_{i+1} \in \mathbb{R}^{n}$ given by expression (20)

1 nor $=\|J\|_{\infty} \Delta t$

$2 j_{J \Delta t}=\max \left(0,1+\operatorname{int}\left(\log _{2}(\right.\right.$ nor $\left.\left.)\right)\right) ; s=\frac{\Delta t}{2^{j} J \Delta t} ; J=s J$ 
$3 X_{11}=J ; X_{12}=s I_{n}$

$4 N_{11}=I_{n}+c_{1}(1) J ; N_{12}=c_{1}(1) s I_{n}$

$5 D_{11}=I_{n}+c_{2}(1) J ; D_{12}=c_{2}(1) s I_{n}$

6 For $k=2: q$

$6.1 X_{12}=s X_{11} ; X_{11}=X_{11} J$

6.2 $N_{11}=N_{11}+c_{1}(k) X_{11} ; N_{12}=N_{12}+c_{1}(k) X_{12}$

$6.3 D_{11}=D_{11}+c_{2}(k) X_{11} ; D_{12}=D_{12}+c_{2}(k) X_{12}$

7 Solve $D_{11} F_{11}=N_{11}$ for $F_{11}$

8 Solve $D_{11} F_{12}=N_{12}-D_{12}$ for $F_{12}$

9 For $k=1: j_{J \Delta t}$

$9.1 F_{12}=F_{11} F_{12}+F_{12}$

9.2 $F_{11}=F_{11}^{2}$

$9.3 s=2 s$

$10 y_{i+1}=y_{i}+F_{12} f$

\subsection{Algorithms not based on scale-squaring technique}

Since matrix $C$ of Algorithm 2 is multiplied by $\Delta t$, it is possible compute the approximate solution $x_{i+1}$ without using the scaling-squaring technique. Therefore, the computational costs are reduced without loss of accuracy (see Section 4). Note that in this case it not is necessary to compute block $N_{11}$. The following algorithms solve IVPs for ODEs by that method:

- Algorithm 9 (inolwp) solves the IVP for non-autonomous ODEs (1) by a piecewise-linearized approach without scaling-square of the diagonal Padé approximants.

- Algorithm 10 (iaolwp) solves the IVP for autonomous ODEs (1) by a piecewise-linearized approach without scaling-square of the diagonal Padé approximants.

These algorithms use the following auxiliary algorithms:

- Algorithm 11 (inlbwp) computes the approximate solution at $t_{i+1}$ of IVP for non-autonomous ODEs (1), obtained after the piecewise-linearized process, by a block-oriented implementation without scaling-squaring of diagonal Padé method. The approximate computational cost of Algorithm 11 is $2\left(q+\frac{4}{3}\right) n^{3}$ flops.

- Algorithm 12 (ialbwp) computes the approximate solution at $t_{i+1}$ of IVP for autonomous ODEs (1), obtained after the piecewise-linearized process, by a block-oriented version without scaling-squaring implementation of the diagonal Padé method. The approximate computational cost of this algorithm is $2\left(q+\frac{1}{3}\right) n^{3}$ flops.

Figure 1 shows a scheme with the developed piecewise-linearized algorithms. 
Algorithm $9\left\{x_{i}\right\}=\operatorname{inolwp}\left(\right.$ data $\left., x_{0}, t_{0}, t_{f}, \Delta t, q\right)$

Inputs: Data is a function that computes $f(\tau, x) \in \mathbb{R}^{n}, J(\tau, x) \in \mathbb{R}^{n \times n}$ and $g(\tau, x) \in \mathbb{R}^{n}\left(\tau \in \mathbb{R}, x \in \mathbb{R}^{n}\right)$; vector $x_{0} \in \mathbb{R}^{n}$ of initial conditions; initial time $t_{0} \in \mathbb{R} ;$ final time $t_{f} \in \mathbb{R} ;$ step size $\Delta t \in \mathbb{R} ;$ order $q \in \mathbb{N}$ of the diagonal Padé approximation of the exponential function

Outputs: Vector of solutions $\left\{x_{i}\right\}\left(x_{i} \in \mathbb{R}^{n}\right)$ at $t_{0}, t_{0}+\Delta t, t_{0}+2 \Delta t, \ldots$

$1\left[c_{1}, c_{2}\right]=\operatorname{coedpa}(q)$ (Algorithm 5)

$2 m=\left\lceil\left(t_{f}-t_{0}\right) / \Delta t\right\rceil$

3 For $i=0: m-1$

$3.1[f, J, g]=\operatorname{data}\left(t_{i}, x_{i}\right)$

3.2 $x_{i+1}=\operatorname{inlbwp}\left(J, f, g, x_{i}, \Delta t, c_{1}, c_{2}\right)$ (Algorithm 11)

$3.3 t_{i+1}=t_{i}+\Delta t$

Algorithm $10\left\{x_{i}\right\}=$ iaolwp $\left(\right.$ data $\left., x_{0}, t_{0}, t_{f}, \Delta t, q\right)$

Inputs: data is a function that computes $f(\tau, x) \in \mathbb{R}^{n}$ and $J(\tau, x) \in \mathbb{R}^{n \times n}$ $\left(\tau \in \mathbb{R}, x \in \mathbb{R}^{n}\right)$; vector $x_{0} \in \mathbb{R}^{n}$ of initial conditions; initial time $t_{0} \in \mathbb{R} ;$ final time $_{f} \in \mathbb{R} ;$ step size $\Delta t \in \mathbb{R} ;$ order $q \in \mathbb{N}$ of the diagonal Padé approximation of the exponential function

Outputs: Vector of solutions $\left\{x_{i}\right\}\left(x_{i} \in \mathbb{R}^{n}\right)$ at $t_{0}, t_{0}+\Delta t, t_{0}+2 \Delta t, \ldots$

$1\left[c_{1}, c_{2}\right]=\operatorname{coedpa}(q)$ (Algorithm 5)

$2 m=\left\lceil\left(t_{f}-t_{0}\right) / \Delta t\right\rceil$

3 For $i=0: m-1$

$3.1[f, J, g]=\operatorname{data}\left(t_{i}, x_{i}\right)$

3.2 $x_{i+1}=\operatorname{ialbwp}\left(J, f, x_{i}, \Delta t, c_{1}, c_{2}\right)$ (Algorithm 12)

$3.3 t_{i+1}=t_{i}+\Delta t$

Algorithm $11 y_{i+1}=\operatorname{inlbwp}\left(J, f, g, y_{i}, \Delta t, c_{1}, c_{2}\right)$

Inputs: Jacobian matrix $J \in \mathbb{R}^{n \times n}$; function vector $f \in \mathbb{R}^{n}$; gradient vector $g \in \mathbb{R}^{n}$; vector $y_{i} \in \mathbb{R}^{n}$; step size $\Delta t \in \mathbb{R}$; vectors $c_{1}, c_{2} \in \mathbb{R}^{q}$ with the coefficients of terms of degree greater than 0 in the $(q, q)$ diagonal Padé approximation of the exponential function

Output: Vector $y_{i+1} \in \mathbb{R}^{n}$ given by expression (18)

$1 J=\Delta t J$

$2 X_{11}=J ; X_{12}=\Delta t I_{n} ; X_{13}=0_{n}$

$3 N_{12}=c_{1}(1) I_{n} ; N_{13}=0_{n}$

$4 D_{11}=I_{n}+c_{2}(1) J ; D_{12}=c_{2}(2) s I_{n} ; D_{13}=0_{n}$

5 For $k=2: q$

$5.1 X_{13}=s X_{12} ; X_{12}=s X_{11} ; X_{11}=X_{11} J$

5.2 $N_{12}=N_{12}+c_{1}(k) X_{12} ; N_{13}=N_{13}+c_{1}(k) X_{13}$

$5.3 D_{11}=D_{11}+c_{2}(k) X_{11} ; D_{12}=D_{12}+c_{2}(k) X_{12} ; D_{13}=D_{13}+c_{2}(k) X_{13}$

6 Solve $D_{11} F_{12}=N_{12}-D_{12}$ for $F_{12}$

7 Solve $D_{11} F_{13}=N_{13}-s D_{12}-D_{13}$ for $F_{13}$ 


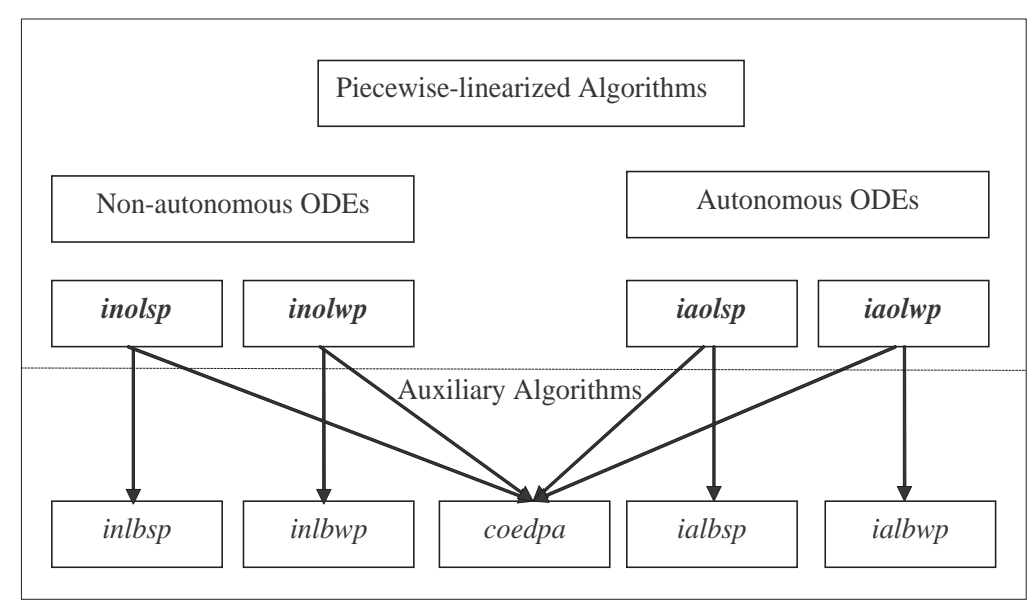

Fig. 1. Schema of piecewise-linearized Algorithms 4(inolsp), 9(inolwp), 7(iaolsp) and $10($ iaolwp $)$

$$
8 y_{i+1}=y_{i}+F_{12} f+F_{13} g
$$

Algorithm $12 y_{i+1}=\operatorname{ialbwp}\left(J, f, y_{i}, \Delta t, c_{1}, c_{2}\right)$

Inputs: Jacobian matrix $J \in \mathbb{R}^{n \times n}$; function vector $f \in \mathbb{R}^{n}$; vector $y_{i} \in \mathbb{R}^{n}$; step size $\Delta t \in \mathbb{R}$; vectors $c_{1}, c_{2} \in \mathbb{R}^{q}$ with the coefficients of terms of degree greater than 0 in the $(q, q)$ diagonal Padé approximation of the exponential function

Output: Vector $y_{i+1} \in \mathbb{R}^{n}$ given by expression (20)

$$
\begin{array}{rl}
1 & J=\Delta t J \\
2 & X_{11}=J ; X_{12}=\Delta t I_{n} \\
3 & D_{11}=I_{n}+c_{2}(1) J \\
4 & D_{12}=c_{2}(1) s I_{n} \\
5 & \text { For } k=2: q \\
& 5.1 X_{12}=s X_{11} ; X_{11}=X_{11} J \\
& 5.2 N_{12}=N_{12}+c_{1}(k) X_{12} \\
& 5.3 D_{11}=D_{11}+c_{2}(k) X_{11} ; D_{12}=D_{12}+c_{2}(k) X_{12} \\
6 & \text { Solve } D_{11} F_{12}=N_{12}-D_{12} \text { for } F_{12} \\
7 & y_{i+1}=y_{i}+F_{12} f
\end{array}
$$

\section{$4 \quad$ Experimental results}

The main objective of this section is to compare the algorithms developed in Sections 2 and 3. What follows is a short description of the characteristic parameters for the implemented algorithms:

- iaolsp and inolsp solve IVPs for ODEs by means of a piecewise-linearized approach and a block-oriented version with scaling-squaring implementation 
of the diagonal Padé approximation method:

- Order $(q)$ of the diagonal Padé approximation of the exponential function.

- iaolwp and inolwp solve IVPs for ODEs by means of a piecewise-linearized approach and a block-oriented version without scaling-squaring implementation of the diagonal Padé approximation method:

- Order $(q)$ of the diagonal Padé approximation of the exponential function.

- iodbcs solves IVPs for ODEs by means of a BDF method based on a ChordShamanskii iteration:

- Order $(r)$ of BDF method.

- Relative error tolerance $\left(t o l_{1}\right)$ and absolute error tolerance $\left(t o l_{2}\right)$.

- Maximum number of iterations without computing the Jacobian matrix $(m)$.

- threshold $(\rho)$.

As test battery five case studies were considered. The criteria to select these cases studies were:

- To solve physics and physical chemistry problems by the developed algorithms (case studies 1, 2, 3 and 5). Case study 4 has been selected because it has a known analytic solution.

- To prove the implementations on autonomous and non-autonomous ODEs: three IVPs for autonomous ODEs and two IVPs for non-autonomous ODEs have been selected.

- To compare the implementations when the ODE is stiff (cases studies 1, 2, 3 and 5) or non-stiff (case study 4).

Numerous tests were made (for each case study the characteristic parameters were varied, although only the parameters which offered better accuracy and lower computational cost for each algorithm are presented). Three kinds of tests are shown:

- Variable step size.

- Variable final time.

For each test, the following results are shown:

- Tables which contain the relative error

$$
E_{r}=\frac{\left\|x-x^{*}\right\|_{\infty}}{\|x\|_{\infty}}
$$

where $x^{*}$ is the computed solution and $x$ is the analytic solution (case study 4 ) or the solution computed by the MATLAB function ode15s with a vector of relative error tolerances rtol $=10^{-13}$ and a vector of absolute error tolerances atol $=10^{-13}[14]$ (case studies 1, 2, 3 and 5).

- Tables with the execution time. 
All algorithms were implemented in MATLAB and Fortran. The MATLAB implementations were tested on an Intel Core 2 Duo processor at $1.83 \mathrm{GHz}$ with 2 GB main memory, using MATLAB version 7.5. The tests for the larger dimension problem (case study 5) were carried out on a SGI Altix 3700 node [15], with $1.3 \mathrm{GHz}$ Intel Itanium II with $3 \mathrm{MB}$ cache. In this case the algorithms were implemented in FORTRAN using the mathematical libraries BLAS [16] and LAPACK [17]. The implementations were compiled with Intel FORTRAN compiler (release 8.1) and SGI SCSL (Scientific Computing Software Library) mathematical library (release 1.5.1) was used. The SCSL is an optimized version of BLAS and LAPACK for SGI systems. The implemented algorithms are available online at [18]. The case studies considered in this work are presented below.

\subsection{Case study 1 (Chemical Akzo Nobel problem)}

This case study corresponds to the stiff autonomous ODE [19] defined by

$$
\begin{array}{r}
\dot{x}=f(x), x=x(t) \in \mathbb{R}^{6}, 0 \leq t \leq 180, \\
x(0)=\left[\begin{array}{lll}
0.444, & 0.00123,0,0,0.007, & 0.35999964
\end{array}\right]^{T},
\end{array}
$$

where

$$
f(x)=\left[\begin{array}{c}
-2 r_{1}+r_{2}-r_{3}-r_{4} \\
-0.5 r_{1}-r_{4}-0.5 r_{5}+F_{i n} \\
r_{1}-r_{2}+r_{3} \\
-r_{2}-r_{3}-2 r_{4} \\
r_{2}-r_{3}+2 r_{5} \\
-r_{5}
\end{array}\right]
$$

and $r_{i}, i=1,2,3,4,5$, and $F_{\text {in }}$ are defined as

$$
\begin{gathered}
r_{1}=k_{1} x_{1}^{4} x_{2}^{0.5}, \\
r_{2}=k_{2} x_{3} x_{4}, \\
r_{3}=\frac{k_{2}}{K} x_{1} x_{5}, \\
r_{4}=k_{3} x_{1} x_{4}^{2}, \\
r_{5}=k_{4} x_{6}^{2} x_{2}^{0.5}, \\
F_{\text {in }}=k l A\left(\frac{p\left(\mathrm{CO}_{2}\right)}{H}-x_{2}\right) .
\end{gathered}
$$


Table 2

\begin{tabular}{cccccc}
\hline Er & $\Delta t=0.1$ & $\Delta t=0.05$ & $\Delta t=0.01$ & $\Delta t=0.005$ & $\Delta t=0.001$ \\
\hline iodbcs & $2.576 \mathrm{e}-5$ & $1.223 \mathrm{e}-5$ & $8.658 \mathrm{e}-7$ & $2.323 \mathrm{e}-7$ & $9.823 \mathrm{e}-9$ \\
\hline iaolsp & $2.080 \mathrm{e}-5$ & $5.238 \mathrm{e}-6$ & $1.485 \mathrm{e}-7$ & $3.851 \mathrm{e}-8$ & $1.588 \mathrm{e}-9$ \\
\hline iaolwp & $8.100 \mathrm{e}-6$ & $2.824 \mathrm{e}-6$ & $1.485 \mathrm{e}-7$ & $3.851 \mathrm{e}-8$ & $1.588 \mathrm{e}-9$ \\
\hline
\end{tabular}

Relative errors considering $t_{f}=60$ and varying $\Delta t$ (case study 1 )

The tests were carried out considering:

$$
\begin{aligned}
& k_{1}=18.7, \quad K=34.4, \\
& k_{2}=0.58, \quad k l A=3.3, \\
& k_{3}=0.09, \quad p\left(\mathrm{CO}_{2}\right)=0.9 \\
& k_{4}=0.42, \quad H=737 .
\end{aligned}
$$

This problem originates from Akzo Nobel Central Research in Arnhem. It describes a chemical process in which two species, FLB and ZHU, are mixed, while carbon dioxide is continuously added. The variables $x_{i}$ correspond to the following concentrations: $x_{1}=[\mathrm{FLB}], x_{2}=\left[\mathrm{CO}_{2}\right], x_{3}=[\mathrm{FLBT}], x_{4}=[\mathrm{ZHU}]$, $x_{5}=[\mathrm{ZLA}]$ and $x_{6}=[\mathrm{ZLA} . \mathrm{ZHU}]$, where FLBT, ZLA and ZLA.ZHU are other species that appear in the chemical process.

The optimal values of characteristic parameters were:

- iodbcs: $r=3$, tol $_{1}=$ tol $_{2}=10^{-14}, m=2, \rho=0.5$.

- iaolsp: $q=1$.

- iaolwp: $q=1$.

The following tests were done:

- First test (Tables 2 and 3 ): $t_{f}=60$ and $\Delta t$ variable.

- Second test (Table 4 and Figure 2): $\Delta t=0.01$ and $t_{f}$ variable.

Conclusions for this case study:

- Considering the same step size, the implementations based on the piecewiselinearized approach have lower relative error and lower execution time than the implementation based on BDF approach.

- The implementation based on the piecewise-linearized method and on the diagonal Padé approach without scaling-squaring (iaolwp) has the shorter execution time. 


\begin{tabular}{cccccc}
\hline Execution time (seconds) & $\Delta t=0.1$ & $\Delta t=0.05$ & $\Delta t=0.01$ & $\Delta t=0.005$ & $\Delta t=0.001$ \\
\hline iodbcs & 0.196 & 0.253 & 1.275 & 2.545 & 11.43 \\
\hline iaolsp & 0.087 & 0.153 & 0.745 & 1.493 & 7.462 \\
\hline iaolwp & 0.058 & 0.114 & 0.570 & 1.138 & 5.692 \\
\hline
\end{tabular}

Table 3

Execution time of the MATLAB implementations considering $t_{f}=60$ and varying $\Delta t$ (case study 1 )

\begin{tabular}{cccccc}
\hline$E r$ & $t_{f}=60$ & $t_{f}=90$ & $t_{f}=120$ & $t_{f}=150$ & $t_{f}=180$ \\
\hline iodbcs & $8.658 \mathrm{e}-7$ & $5.388 \mathrm{e}-7$ & $4.183 \mathrm{e}-7$ & $3.607 \mathrm{e}-7$ & $3.303 \mathrm{e}-7$ \\
\hline iaolsp & $1.485 \mathrm{e}-7$ & $9.687 \mathrm{e}-8$ & $7.838 \mathrm{e}-8$ & $6.980 \mathrm{e}-8$ & $6.546 \mathrm{e}-8$ \\
\hline iaolwp & $1.485 \mathrm{e}-7$ & $9.687 \mathrm{e}-8$ & $7.838 \mathrm{e}-8$ & $6.980 \mathrm{e}-8$ & $6.546 \mathrm{e}-8$ \\
\hline
\end{tabular}

Table 4

Relative errors considering $\Delta t=0.01$ and varying $t_{f}$ (case study 1 )

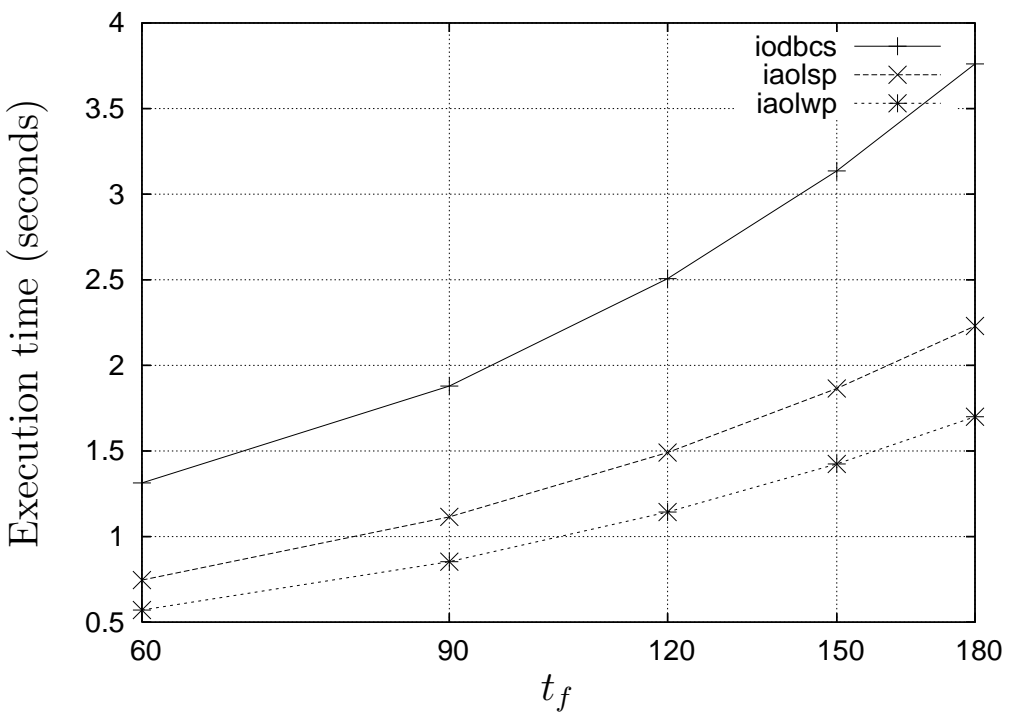

Fig. 2. Execution time of the MATLAB implementations considering $\Delta t=0.01$ and varying $t_{f}$ (case study 1 )

\subsection{Case study 2 (HIRES problem)}

This case study is presented in [19] and it corresponds to the stiff IVP for ODEs defined by

$$
\begin{gathered}
\dot{x}=f(x), x=x(t) \in \mathbb{R}^{8}, 0 \leq t \leq 321.8122, \\
x(0)=[1,0,0,0,0,0,0,0.0057]^{T},
\end{gathered}
$$




\begin{tabular}{cccccc}
\hline$E r$ & $\Delta t=0.1$ & $\Delta t=0.05$ & $\Delta t=0.01$ & $\Delta t=0.005$ & $\Delta t=0.001$ \\
\hline iodbcs & $2.136 \mathrm{e}-4$ & $5.279 \mathrm{e}-5$ & $1.933 \mathrm{e}-6$ & $4.767 \mathrm{e}-7$ & $1.885 \mathrm{e}-8$ \\
\hline iaolsp & $4.185 \mathrm{e}-5$ & $1.147 \mathrm{e}-5$ & $4.8495 \mathrm{e}-7$ & $1.219 \mathrm{e}-7$ & $4.899 \mathrm{e}-9$ \\
\hline iaolwp & $4.183 \mathrm{e}-5$ & $1.147 \mathrm{e}-5$ & $4.8495 \mathrm{e}-7$ & $1.219 \mathrm{e}-7$ & $4.899 \mathrm{e}-9$ \\
\hline
\end{tabular}

Table 5

Relative errors considering $t_{f}=50$ and varying $\Delta t$ (case study 2 )

where

$$
f(x)=\left(\begin{array}{c}
-1.71 x_{1}+0.43 x_{2}+8.32 x_{3}+0.0007 \\
1.71 x_{1}-8.75 x_{2} \\
-10.03 x_{3}+0.43 x_{4}+0.035 x_{5} \\
8.32 x_{2}+1.71 x_{3}-1.12 x_{4} \\
-1.745 x_{5}+0.43 x_{6}+0.43 x_{7} \\
-280 x_{6} x_{8}+0.69 x_{4}+1.71 x_{5}-0.43 x_{6}+0.69 x_{7} \\
280 x_{6} x_{8}-1.81 x_{7} \\
-280 x_{6} x_{8}+1.81 x_{7}
\end{array}\right) .
$$

The name HIRES was given by Hairer and Wanner [1]. The HIRES problem explains the "High Irradiance Responses" (HIRES) of phytochrome-mediated photomorphogenesis by means of a chemical reaction involving eight reactants. The variables $x_{i}$ are the concentrations of the eight reactants.

The optimal values of characteristic parameters were:

- iodbcs: $r=3$, tol $_{1}=$ tol $_{2}=10^{-14}, m=2, \rho=0.5$.

- iaolsp: $q=2$.

- iaolwp: $q=2$.

The following tests were done:

- First test (Tables 5 and 6$): t_{f}=50$ and $\Delta t$ variable.

- Second test (Table 7 and Figure 3): $\Delta t=0.01$ and $t_{f}$ variable.

The conclusions obtained in this case are the same as for case study 1 . 


\begin{tabular}{cccccc}
\hline Execution time (seconds) & $\Delta t=0.1$ & $\Delta t=0.05$ & $\Delta t=0.01$ & $\Delta t=0.005$ & $\Delta t=0.001$ \\
\hline iodbcs & 0.199 & 0.300 & 1.521 & 2.471 & 10.258 \\
\hline iaolsp & 0.137 & 0.250 & 0.991 & 1.819 & 9.098 \\
\hline iaolwp & 0.069 & 0.139 & 0.709 & 1.316 & 6.578 \\
\hline
\end{tabular}

Table 6

Execution time of the MATLAB implementations considering $t_{f}=50$ and varying $\Delta t$ (case study 2)

\begin{tabular}{cccccc}
\hline Er & $t_{f}=100$ & $t_{f}=150$ & $t_{f}=200$ & $t_{f}=250$ & $t_{f}=300$ \\
\hline iodbcs & $2.294 \mathrm{e}-6$ & $2.989 \mathrm{e}-6$ & $4.276 \mathrm{e}-6$ & $7.425 \mathrm{e}-6$ & $2.406 \mathrm{e}-5$ \\
\hline iaolsp & $5.753 \mathrm{e}-7$ & $7.496 \mathrm{e}-7$ & $1.072 \mathrm{e}-6$ & $1.862 \mathrm{e}-6$ & $6.041 \mathrm{e}-6$ \\
\hline iaolwp & $5.753 \mathrm{e}-7$ & $7.496 \mathrm{e}-7$ & $1.072 \mathrm{e}-6$ & $1.862 \mathrm{e}-6$ & $6.041 \mathrm{e}-6$ \\
\hline
\end{tabular}

Table 7

Relative errors considering $\Delta t=0.01$ and varying $t_{f}$ (case study 2 )

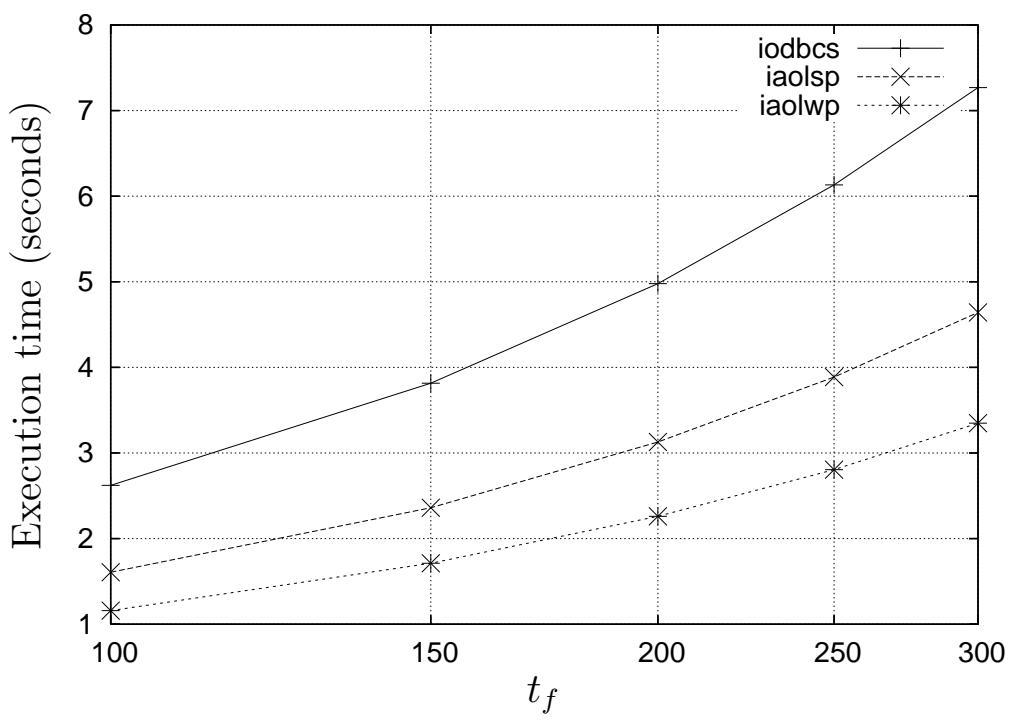

Fig. 3. Execution time of the MATLAB implementations considering $\Delta t=0.01$ and varying $t_{f}$ (case study 2 )

\subsection{Case study 3}

This case study corresponds to the stiff autonomous ODE [14, pp. 29] defined by

$$
\begin{gathered}
\dot{x}=f(x), x=x(t) \in \mathbb{R}^{3}, 0 \leq t \leq 8 \cdot 10^{5}, \\
x(0)=[0,1,0]^{T},
\end{gathered}
$$


where

$$
f\left(\left[x_{1}, x_{2}, x_{3}\right]^{T}\right)=\left(\begin{array}{c}
-k_{1} x_{1}+k_{2} x_{3} \\
-k_{4} x_{2}+k_{3} x_{3} \\
k_{1} x_{1}+k_{4} x_{2}-\left(k_{1}+k_{3}\right) x_{3}
\end{array}\right)
$$

and

$$
\begin{gathered}
k_{1}=8.4303270 \cdot 10^{-10}, k_{2}=2.9002673 \cdot 10^{11}, \\
k_{3}=2.4603642 \cdot 10^{10}, k_{4}=8.7600580 \cdot 10^{-6} .
\end{gathered}
$$

This case study corresponds to the proton transfer hydrogen-hydrogen bond problem, where $x_{1}(t)$ and $x_{2}(t)$ are the solution components of the proton transfer and $x_{3}(t)$ is the quickly reacting intermediate component.

The optimal values of characteristic parameters were:

- iodbcs: $r=2$, tol $_{1}=$ tol $_{2}=10^{-14}, m=2, \rho=0.5$.

- iaolsp: $q=1$.

- iaolsp: $q=1$.

The following tests were done:

- First test (Table 8 and Figure 9): $t_{f}=100$ and $\Delta t$ variable. Since with small values of $\Delta t$ very high precisions have been reached, only $\Delta t=0.1$ and $\Delta t=0.05$ have been considered.

- Second test (Table 10 and Figure 4): $\Delta t=0.01$ and $t_{f}$ variable.

Conclusions for this case study:

- For the same step size, the relative errors committed by the implementation based on the piecewise-linearized method and on the diagonal Padé approach without scaling-squaring (iaolwp) have been minor in comparison to those committed by the other two implementations; also this implementation has the shortest execution time.

- The implementation based on the BDF method (iodbcs) has a lower relative error than the implementation based on the piecewise-linearized method and on the diagonal Padé approach with scaling-squaring (iaolsp), but execution time is longer. 
Table 8

\begin{tabular}{ccc}
\hline Er & $\Delta t=0.1$ & $\Delta t=0.05$ \\
\hline iodbcs & $4.104 \mathrm{e}-14$ & $8.202 \mathrm{e}-14$ \\
\hline iaolsp & $4.706 \mathrm{e}-12$ & $2.358 \mathrm{e}-12$ \\
\hline iaolwp & $6.274 \mathrm{e}-15$ & $6.065 \mathrm{e}-15$
\end{tabular}

Relative errors considering $t_{f}=100$ and varying $\Delta t$ (case study 3 )

Table 9

\begin{tabular}{ccc}
\hline Execution time (seconds) & $\Delta t=0.1$ & $\Delta t=0.05$ \\
\hline iodbcs & 0.199 & 0.300 \\
\hline iaolsp & 0.137 & 0.250 \\
\hline iaolwp & 0.069 & 0.139 \\
\hline
\end{tabular}

Execution time of the MATLAB implementations considering $t_{f}=100$ and varying $\Delta t$ (case study 3 )

Table 10

\begin{tabular}{cccccc}
\hline Er & $t_{f}=500$ & $t_{f}=1000$ & $t_{f}=1500$ & $t_{f}=2000$ & $t_{f}=2500$ \\
\hline iodbcs & $4.359 \mathrm{e}-12$ & $8.787 \mathrm{e}-12$ & $1.420 \mathrm{e}-12$ & $1.995 \mathrm{e}-11$ & $2.6616 \mathrm{e}-11$ \\
\hline iaolsp & $4.636 \mathrm{e}-12$ & $9.071 \mathrm{e}-12$ & $1.538 \mathrm{e}-12$ & $2.130 \mathrm{e}-11$ & $2.666 \mathrm{e}-11$ \\
\hline iaolwp & $3.838 \mathrm{e}-14$ & $1.303 \mathrm{e}-13$ & $1.927 \mathrm{e}-12$ & $3.960 \mathrm{e}-12$ & $5.814 \mathrm{e}-12$ \\
\hline
\end{tabular}

Relative errors considering $\Delta t=0.01$ and varying $t_{f}$ (case study 3 )

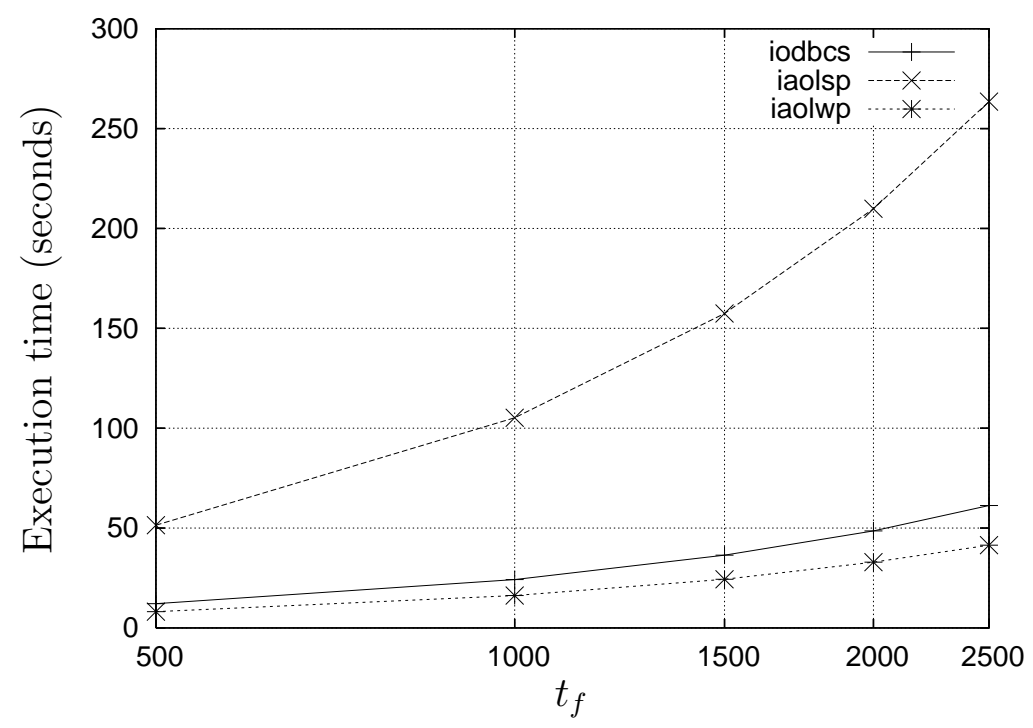

Fig. 4. Execution time of the MATLAB implementations considering $\Delta t=0.01$ and varying $t_{f}$ (case study 3 ) 


\begin{tabular}{cccccc}
\hline$E r$ & $\Delta t=0.1$ & $\Delta t=0.05$ & $\Delta t=0.01$ & $\Delta t=0.005$ & $\Delta t=0.001$ \\
\hline iodbcs & $5.167 \mathrm{e}-06$ & $1.171 \mathrm{e}-06$ & $4.103 \mathrm{e}-08$ & $1.009 \mathrm{e}-08$ & $3.971 \mathrm{e}-10$ \\
\hline inolsp & $1.079 \mathrm{e}-15$ & $1.447 \mathrm{e}-15$ & $6.296 \mathrm{e}-15$ & $2.268 \mathrm{e}-14$ & $4.965 \mathrm{e}-14$ \\
\hline inolwp & $1.079 \mathrm{e}-15$ & $1.447 \mathrm{e}-15$ & $6.296 \mathrm{e}-15$ & $2.268 \mathrm{e}-14$ & $4.965 \mathrm{e}-14$ \\
\hline
\end{tabular}

Table $\overline{11}$

Relative errors considering $t_{f}=10$ and varying $\Delta t$ (case study 4 )

\subsection{Case study 4}

This case study corresponds to the non-stiff and non-autonomous ODE [6] defined as

$$
\begin{gathered}
\dot{x}(t)=(t-x(t))^{2}+1, t \geq 3, \\
x(3)=2 .
\end{gathered}
$$

The analytic solution is

$$
x(t)=t+\frac{1}{2-t} .
$$

The values chosen for the characteristic parameters were:

- iodbcs: $r=2$, tol $_{1}=$ tol $_{2}=10^{-12}, m=2, \rho=0.5$.

- inolsp: $q=1$.

- inolwp: $q=1$.

The following tests were done:

- First test (Tables 11 and 12): $t_{f}=10$ and $\Delta t$ variable. Since the piecewiselinearized method presents a very small error for a step size equal to 0.1, we will show only the results obtained for that increase.

- Second test (Table 13 and Figure 5): $\Delta t=0.1$ and $t_{f}$ is variable.

Conclusions for this case study:

- For the same step size, the implementation based on the BDF method iodbcs shows more significant error than the implementations based on the piecewise-linearized method.

- The implementation based on the piecewise-linearized method and on the diagonal Padé approach without scaling-squaring (inolwp) has the shortest execution time. 


\begin{tabular}{cccccc}
\hline Execution time (seconds) & $\Delta t=0.1$ & $\Delta t=0.05$ & $\Delta t=0.01$ & $\Delta t=0.005$ & $\Delta t=0.001$ \\
\hline iodbcs & 0.015 & 0.027 & 0.071 & 0.121 & 0.609 \\
\hline inolsp & 0.010 & 0.017 & 0.046 & 0.092 & 0.456 \\
\hline inolwp & 0.008 & 0.015 & 0.040 & 0.080 & 0.400 \\
\hline
\end{tabular}

Table 12

Execution time the MATLAB implementations for $t_{f}=10$ and $\Delta t$ (case study 4 )

\begin{tabular}{cccccc}
\hline Er & $t_{f}=100$ & $t_{f}=200$ & $t_{f}=300$ & $t_{f}=400$ & $t_{f}=500$ \\
\hline iodbcs & $1.192 \mathrm{e}-08$ & $1.460 \mathrm{e}-09$ & $4.295 \mathrm{e}-10$ & $1.807 \mathrm{e}-10$ & $9.228 \mathrm{e}-11$ \\
\hline inolsp & $1.236 \mathrm{e}-14$ & $1.904 \mathrm{e}-14$ & $1.762 \mathrm{e}-14$ & $5.032 \mathrm{e}-14$ & $6.209 \mathrm{e}-14$ \\
\hline inolwp & $1.236 \mathrm{e}-14$ & $1.904 \mathrm{e}-14$ & $1.762 \mathrm{e}-14$ & $5.032 \mathrm{e}-14$ & $6.209 \mathrm{e}-14$
\end{tabular}

Table $1 \overline{3}$

Relative errors considering $\Delta t=0.1$ and varying $t_{f}$ (case study 4 )

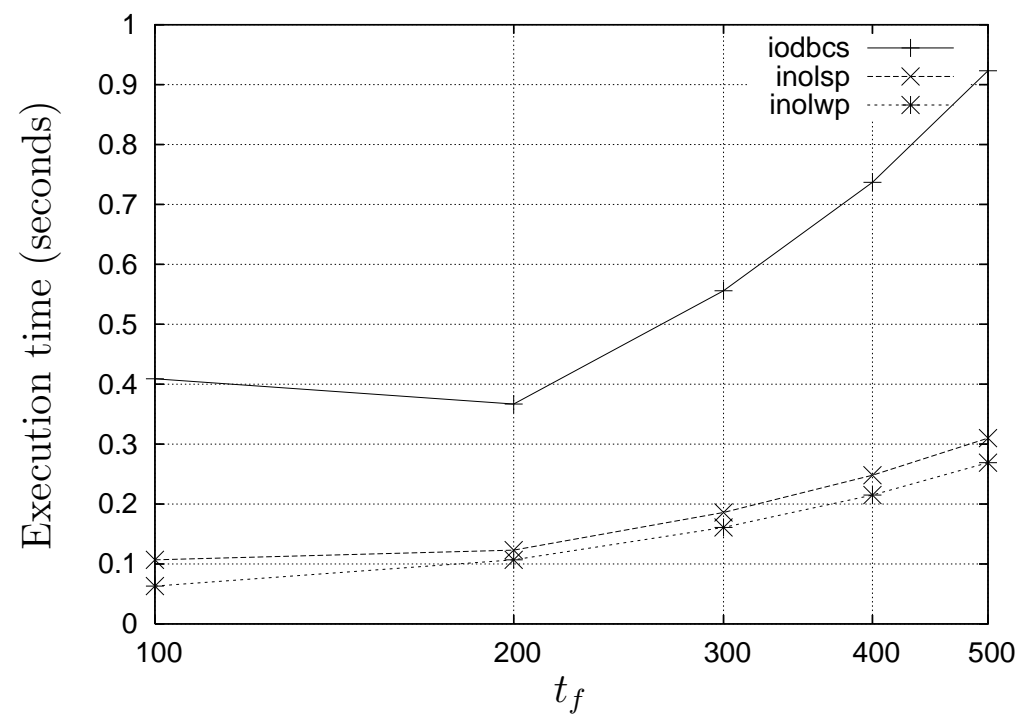

Fig. 5. Execution time of the MATLAB implementations considering $\Delta t=0.1$ and varying $t_{f}$ (case study 4 )

\subsection{Case study 5 (Medical Akzo Nobel problem)}

This case study corresponds to a stiff non-autonomous ODE [19] defined as

$$
\begin{gathered}
\dot{x}=f(t, x), t \geq 0, x=x(t) \in \mathbb{R}^{2 N}, 0 \leq t \leq 20, \\
x(0)=\left[0, v_{0}, 0, v_{0}, \cdots, 0, v_{0}\right] \in \mathbb{R}^{2 N}, N=200 .
\end{gathered}
$$


Function $f$ is defined as follows

$$
\begin{gathered}
f_{2 j-1}=\alpha_{j} \frac{x_{2 j+1}-x_{2 j-3}}{2 \Delta \zeta}+\beta_{j} \frac{x_{2 j-3}-2 x_{2 j-1}+x_{2 j+1}}{(\Delta \zeta)^{2}}-k x_{2 j-1} x_{2 j}, \\
f_{2 j}=-k x_{2 j} x_{2 j-1},
\end{gathered}
$$

where

$$
\begin{aligned}
\alpha_{j} & =\frac{2(j \Delta \zeta-1)^{3}}{c^{2}} \\
\beta_{j} & =\frac{(j \Delta \zeta-1)^{4}}{c^{2}} .
\end{aligned}
$$

The values of $j$ are from 1 to $N, \Delta \zeta=\frac{1}{N}, x_{-1}(t)=\phi(t), x_{2 N+1}=x_{2 N-1}$ and $\phi$ function is given by

$$
\phi(t)=\left\{\begin{array}{l}
2, t \in(0,5] \\
0, t \in(5,20]
\end{array} .\right.
$$

The Akzo Nobel research laboratories formulated this problem in their study of the penetration of radio-labeled antibodies into a tissue infected by a tumor. This study was carried out for diagnostic and therapeutic purposes. The desired results are the evolutions of the concentrations $u$ and $v$ of both elements (the antibodies and the tissue, respectively) along the discretized space in function of time. This problem can be formulated as the above ODE by using the lines method. In this formulation, the data vector is noted as $x$, where elements $x_{2 j-1}, j=1, \ldots, N$, represent concentrations $u$ along the spatial dimension and elements $x_{2 j}, j=1, \ldots, N$, represent concentrations $v$. The chosen values of $k, v_{0}$ and $c$ were 100, 1 and 4 respectively.

The optimal values of characteristic parameters were:

- iodbcs: $r=2$, tol $_{1}=$ tol $_{2}=10^{-14}, m=2, \rho=0.5$.

- inolsp: $q=1$.

- inolwp: $q=1$.

Figure 6 summarizes the execution time of the Fortran implementation of the algorithms considered in this work, considering $\Delta t=10^{-6}$ and varying $t$. The relative error in all algorithms was approximately equal to $10^{-6}$. In this case, the implementation based on the piecewise-linearized method and on the diagonal Padé approach without scaling-squaring (inolwp) has the shortest execution time. 


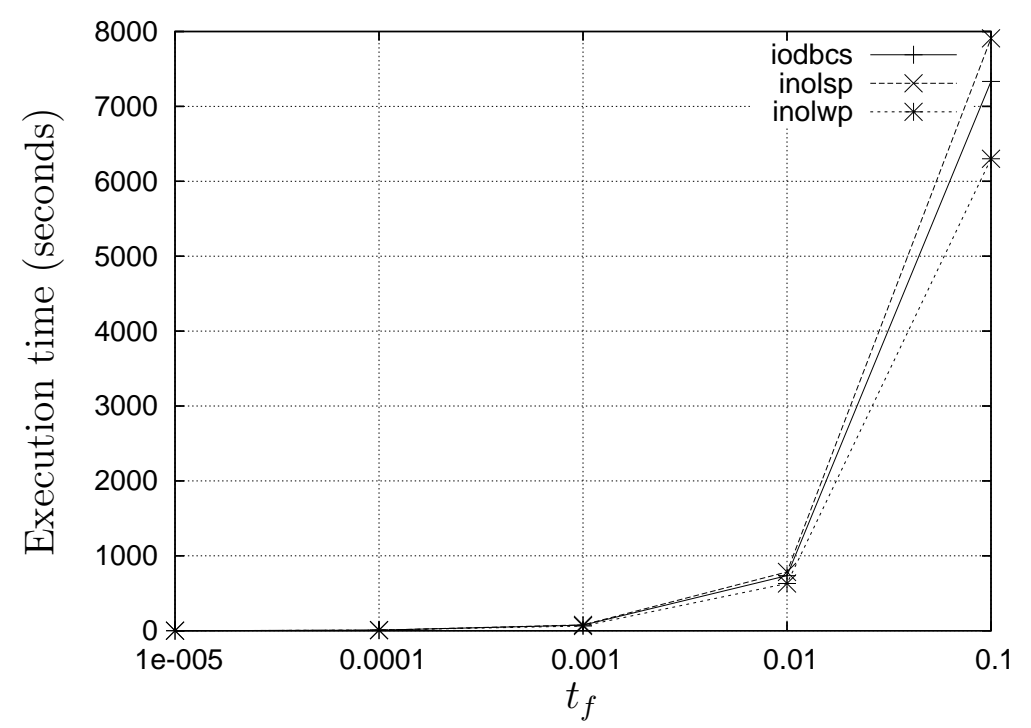

Fig. 6. Execution time of the Fortran implementations considering $\Delta t=10^{-6}$ and varying $t_{f}$ (case study 5 )

\section{Conclusions and future work}

In this paper three methods for solving IVPs for ODEs have been developed and implemented. The first is a BDF method based on a Chord and Shamanskii iteration. The other two methods are based on the piecewise-linearized method and the diagonal Padé approximants. These methods are based on Theorem 1, which makes it possible to solve IVPs for ODEs. In addition, four algorithms, two for non-autonomous ODEs (inolsp-inolwp) and another two for autonomous ODEs (iaolsp-iaolwp), have been implemented. These algorithms have been compared to the BDF algorithm based on Chord-Shamanskii iteration $(i d o b c s)$. According to experimental results, the algorithms based on the piecewise-linearized method and on the diagonal Padé approach without scaling-squaring (iaolwp - inolwp) behave better, both in terms of precision and computational costs, than the BDF algorithm (see Tables 14 and 15). Below is a summary of the five case studies:

(1) The optimal order of the BDF algorithm varies between 1 and 3, depending on the case study.

(2) The optimal order of algorithms based on diagonal Padé approximants varies between 1 (case studies 1, 3, 4 and 5) and 2 (case study 2).

(3) Considering the same step size, the BDF algorithm (idobcs) is generally less accurate than the piecewise-linearized algorithms. The more accurate algorithms are based on the diagonal Padé approximants without scalingsquaring (iaolwp and inolwp algorithms). Also these algorithms have the lowest computational cost.

(4) All implemented algorithms show good behavior in stiff ODEs. 
Table 14

\begin{tabular}{cccccc}
\hline Accuracy & 1-A-S & 2 -A-S & $3-\mathrm{A}-\mathrm{S}$ & 4 NA-NS & 5 NA-S \\
\hline iodbcs & - & - & & - & $\cong$ \\
\hline iaolsp-inolsp & & + & - & + & $\cong$ \\
\hline iaolwp-inolwp & + & + & + & + & $\cong$ \\
\hline
\end{tabular}

Comparative precision of implemented algorithms for the five case studies: The symbols,+- and $\cong$ indicate respectively greater, less and similar precision. A/NA denotes Autonomous/Non-Autonomous ODEs, and S/NS denotes whether the problem is Stiff or Non-Stiff.

Table 15

\begin{tabular}{cccccc}
\hline Execution times & 1-A-S & 2-A-S & 3-A-S & 4 NA-NS & 5 NA-S \\
\hline iodbcs & + & + & & + & \\
\hline iaolsp-inolsp & & & + & & + \\
\hline iaolwp-inolwp & - & - & - & - & - \\
\hline
\end{tabular}

Comparative execution times of the implemented algorithms for the five case studies: The symbols,+- indicate respectively greater and less execution time. A/NA denotes Autonomous/Non-Autonomous ODEs, and S/NS denotes whether the problem is Stiff or Non-Stiff.

As future work, new improvements will be developed, such as:

(1) To consider adapting the methodology described here in order to obtain efficient resolution of ODEs with sparse Jacobian matrices.

(2) To implement algorithms with error control in order to vary step size dynamically. The tests reported here considered constant step size. It is possible to improve the developed algorithms using a step size variable by estimating the error committed in each iteration [10].

(3) To do parallel implementation of the algorithms presented in this work in a distributed memory platform, using the message passing paradigm, MPI [20] and BLACS [21] for communications, and PBLAS [22] and ScaLAPACK [23] for computations.

\section{References}

[1] E. Hairer, G. Wanner, Solving ordinary differential equations II. Stiff and differential-algebraic problems., in: Springer Series in Computational Mathematics, Vol. 14, Springer-Verlag, 1996.

[2] C. F. Curtiss, J. O. Hirschfelder, Integration of stiff equations, in: Proc. Nat. Acad. Sci., Vol. 38, 1952, pp. 235-243. 
[3] A. C. Hindmarsh, Lsode and lsodi, two new initial value ordinary differential equation solvers, ACM-Signum Newslett 15 (1980) 1011.

[4] C. W. Gear, Algorithm 407-difsub for solution of ordinary differential equations, Comm. ACM 14 (1971) 185190.

[5] P. N. Brown, G. D. Byrne, A. C. Hindmarsh, Vode: A variable-coefficient ode solver, SIAM J. Sci. Statist. Comput. 10 (1989) 10381051.

[6] J. I. Ramos, C. M. García-López, Piecewise-linearized methods for initial-value problems, Applied Mathematics and Computation 82 (1997) 273-302.

[7] C. M. García-López, Métodos de linealización para la resolución numérica de ecuaciones diferenciales, Ph.D. thesis, Departamento de Lenguajes y Ciencias de la Computación, Universidad de Málaga (1998).

[8] U. M. Ascher, L. R. Petzold, Computer Methods for Ordinary Differential Equations and Differential-Algebraic Equations, SIAM, 1998.

[9] C. T. Kelley, Iterative Methods for Linear and Nonlinear Equations, Philadelphia, 1995.

[10] C. M. García-López, Piecewise-linearized and linearized $\theta$-methods for ordinary and partial differential equation problems, Computer \& Mathematics with Applications 45 (2003) 351-381.

[11] G. H. Golub, C. V. Loan, Matrix Computations, 3rd Edition, Johns Hopkins Studies in Mathematical Sciences, The Johns Hopkins University Press, 1996.

[12] C. B. Moler, C. V. Loan, Nineteen dubious ways to compute the exponential of a matrix, twenty-five years later*, SIAM Review 45 (2003) 3-49.

[13] N. J. Higham, The scaling and squaring method for the matrix exponential revisited, Tech. Rep. 452, Manchester Centre for Computational Mathematics (2004).

[14] L. S. Shampine, I. Gladwell, S. Thomson, Solving ODEs with MAtlab, Cambridge University Press, 2003.

[15] S. Graphics, SGI Altix Applications Development an Optimization, Release August 1 Edition (2003).

[16] J. Dongarra, J. D. Croz, S. Hammarling, R. J. Hanson, An extended set of FORTRAN Basic Linear Algebra Subroutines, ACM Transactions on Mathematical Software.

[17] E. Anderson, Z. Bai, C. Bischof, J. Demmel, J. Dongarra, J. D. Croz, A. Greenbaum, S. Hammarling, A. McKenney, S. Ostrouchov, D. Sorensen, LAPACK Users' Guide, SIAM, 1992.

[18] http://www.grycap.upv.es/odelin/.

[19] W. M. Lioen, J. J. B. de Swart, Test set for initial value problems solvers, release 2.0 (December 1998). 
[20] W. Gropp, E. Lusk, A. Skjellum, Using MPI: Portable Parallel Programming with the Message-Passing Interface, MIT Press, 1994.

[21] J. J. Dongarra, R. A. V. D. Geijn, Two dimensional basic linear algebra communications subprograms, Tech. rep., Department of Computer Science, University of Tennessee (1991).

[22] J. Choi, J. Dongarra, S. Ostrouchov, A. Petitet, D. Walker, A proposal for a set of parallel basic linear algebra subprogram, Tech. Rep. UT-CS-95-292, Department of Computer Science, University of Tennessee (1995).

[23] L. S. Blackford, J. Choi, A. Cleary, E. D'Azevedo, J. Demmel, I. Dhillon, ScaLAPACK Users' Guide, SIAM, 1997. 